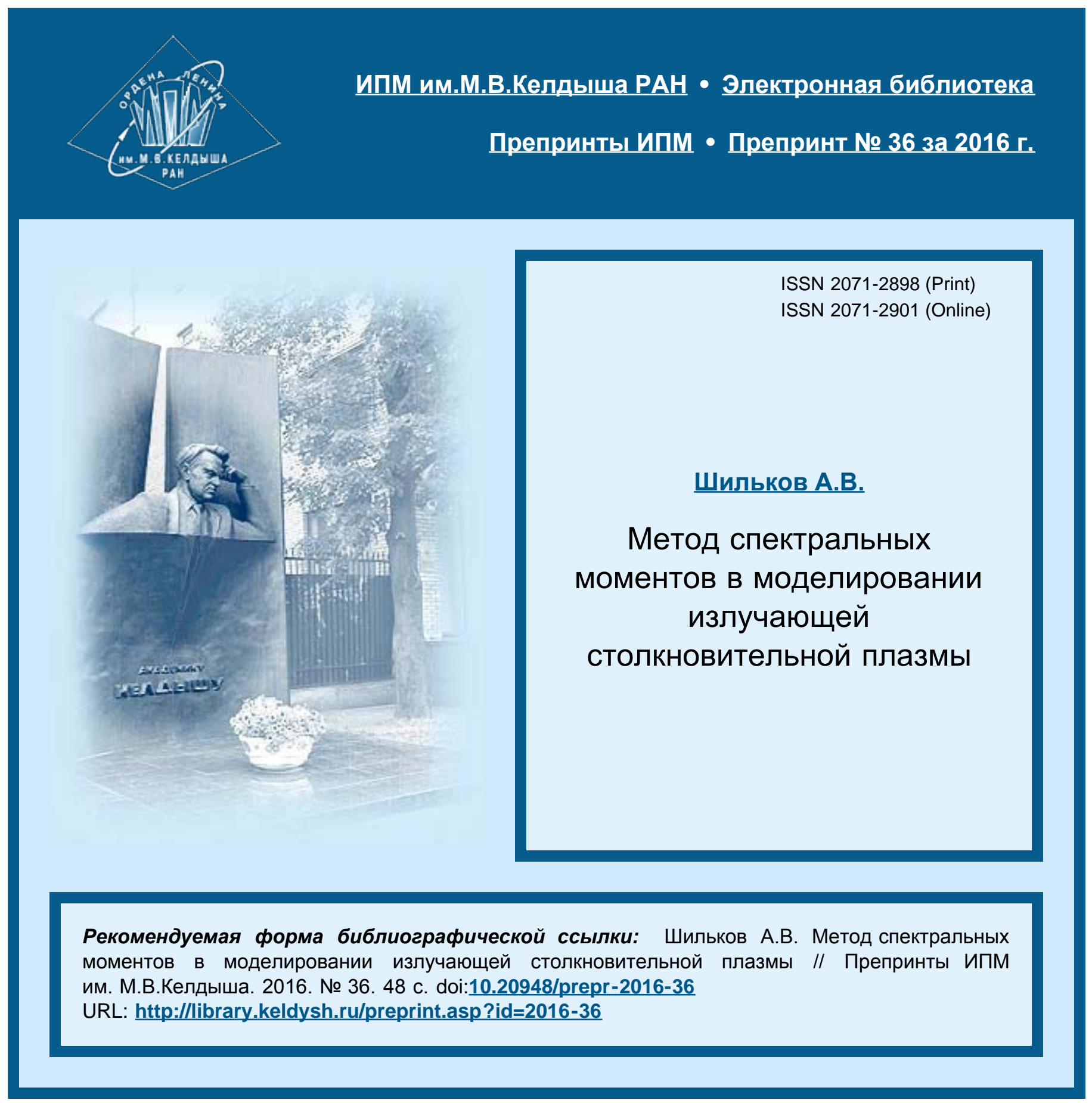




\author{
О рд н н а $Л$ н и на \\ ИНСТИТУТ ПРИКЛАДНОЙ МАТЕМАТИКИ \\ имени М.В.Келдыша \\ Р оссийской а кадемии на ук
}

\begin{abstract}
А.В. Шильков
Метод спектральных моментов

в моделировании

излучающей столкновительной плазмы
\end{abstract}

Москва - 2016 


\section{Шильков А.В.}

\section{Метод спектральных моментов в моделировании излучающей столкновительной плазмы}

Разработан метод спектральных моментов, упрощающий расчет многорезонансных линейчатых спектров излучения неравновесной столкновительной плазмы. Интенсивность излучения раскладывается в ряд по системе базисных функций, которые зависят от энергии фотонов, учитывают резонансную структуру сечений и обеспечивают быструю сходимость разложения в пределах нескольких гармоник. Изложен экономичный способ нахождения коэффициентов разложения - «спектральных моментов» - на основе решения кинетического уравнения для лебегова распределения фотонов. Продемонстрирована быстрая сходимость разложения.

Ключевые слова: перенос излучения, агрегация спектра, восстановление спектра, метод моментов, интеграл Лебега, излучающая плазма

\section{Alexander Victorovich Shilkov \\ Spectral momentum method in collisional-radiative modeling of plasmas}

The spectrum momentum method, simplifying the calculations of multiresonance emission spectra of non-equilibrium collisional plasma, is developed. The intensity of radiation is expanded in a series of basic functions that are dependent on the photon energy, dependent on the resonance structure of the cross sections, and provide a convergence of the expansion in the range of several harmonics. Efficient way of finding the expansion coefficients - "spectral moments" - based on the solution of the kinetic equation for the Lebesgue distribution of photons is described. Fast convergence of the expansion is shown.

Key words: radiative transfer, spectrum aggregation, spectrum recovery, momentum method, Lebesgue integral, collisional-radiative plasmas

Исследование выполнено за счет гранта Российского научного фонда (проект №14-11-00699).

\section{Оглавление}

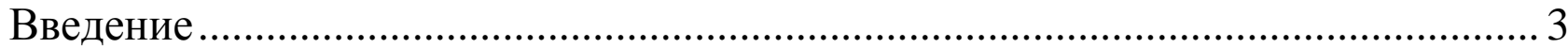

1. Модель радиационно-столкновительной плазмы ..................................... 5

2. Разложение по спектральным моментам ................................................. 20

3. Вычисление спектральных моментов ...................................................2 29

4. Сходимость разложения ............................................................. 33

5. Примерная методика численного решения ............................................. 36

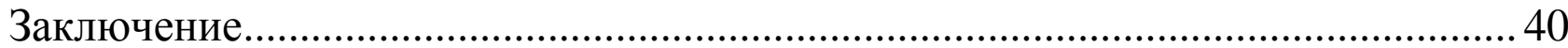

Список литературы........................................................................................ 41

Константы. Обозначения величин ............................................................... 46 


\section{Введение}

Проблема нахождения далекой от равновесия функции распределения фотонов при преимущественно редких столкновениях с частицами вещества, за исключением резонансов поглощения/испускания, возникает в ряде физических исследований и технических приложений. Это создание источников высокочастотного ультрафиолетового излучения, инерциальный термоядерный синтез, диагностика плазмы, исследования сильных ударных волн, моделирование атмосфер звезд и планет, где перенос энергии излучением оказывает существенное влияние на распределение частиц по состояниям возбуждения, на процессы обмена энергией и движение вещества в целом.

Появление достаточно полных банков оцененных расчетноэкспериментальных данных о свойствах атомов, ионов, молекул делает возможным прямое численное моделирование излучающей столкновительной плазмы «из первых принципов». Одна из проблем, которую приходится решать при разработке моделей, связана с наличием множества переходов частиц вещества между квантовыми состояниями и вытекающей отсюда сложной многорезонансной структурой спектра излучения. На Рис. 1 приведено макросечение поглощения излучения парами олова $(\mathrm{Sn})$ при разном разрешении спектра энергий фотонов $E=h v$ (в эB). Олово находится в термодинамическом равновесии при температуре $31.6 э B$ и плотности $1.69 \cdot 10^{-4} 2 / \mathrm{cm}^{3}$. Данные предоставлены В.Г. Новиковым.
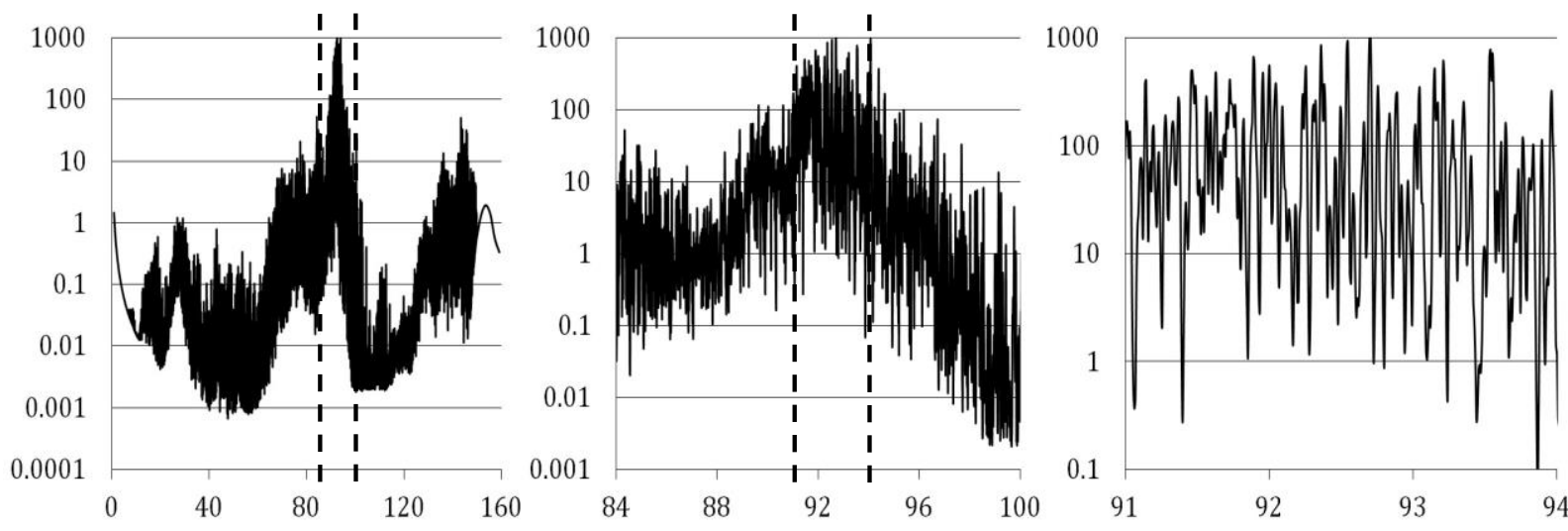

Рис. 1. Макросечение поглощения фотонов $\kappa^{a b}(E)\left(\right.$ в $\left.\mathrm{CM}^{-1}\right)$ оловом (Sn).

Если моделировать спектр фотонов со всеми деталями, то модель оказывается излишне громоздкой. Пока такие модели реализуются в отдельных (benchmark) вычислительных экспериментах на суперкомпьютерах. Они редко используются для проведения серийных инженерных расчетов, требующих варьирования параметров. С другой стороны, если выполнить осреднение, «сглаживание» спектра фотонов, то модель теряет универсальность - либо она применима для частных задач, либо оказывается неточной, либо требует подгонки осредненных констант под результаты натурных экспериментов. 
Цель работы состоит в разработке метода спектральных моментов метода лебеговой агрегации и восстановления детального спектра фотонов по энергии. Метод позволяет экономично и достаточно точно моделировать многорезонансные спектры излучающей столкновительной плазмы без их «сглаживания». Функция распределения фотонов раскладывается в ряд по системе базисных функций, зависящих от энергии фотонов, резонансной структуры сечений и обеспечивающих быструю сходимость разложения. Коэффициенты разложения - спектральные моменты - вычисляются в результате решения кинетического уравнения, сформулированного в терминах функции распределения фотонов на системе лебеговых множеств.

Метод спектральных моментов основан на двух идеях, имеющих свою предысторию. Первая идея состоит в применении интегрирования сильнонемонотонного спектра фотонов по Лебегу вместо интегрирования по Риману. Простой иллюстрацией может служить способ вычисления определенного интеграла от периодической функции в виде интеграла по периоду функции. В этой части данная работа развивает более ранние работы предшественников и автора. Идея разбивать шкалу энергий $0 \leq E<\infty$ на интервалы, соответствующие коридору изменений сечения поглощения частиц $\kappa_{i-1} \leq \kappa(E, r, t)<\kappa_{i}$, и вычислять суммарную скорость поглощения на широком интервале через скорость поглощения на этих интервалах (т.е. по Лебегу) впервые применена в [1]. Затем этот способ неоднократно переоткрывался заново независимыми исследователями в разных формулировках уравнений переноса частиц (фотонов и нейтронов) при разных упрощающих предположениях и под разными названиями [2]-[18]. Более полный перечень работ с кратким пояснением отличий между ними приведен в [19].

В работах автора [14]-[16] дан вывод кинетического уравнения для лебегова распределения частиц (фотонов и нейтронов) из линейного уравнения Больцмана (1) формальным переходом к интегралу Лебега. Метод лебеговского осреднения позволил получить кинетическое уравнение в дифференциальной форме без привязки к способу дискретизации переменных (это важно для разработки метода моментов), выявить недостающие члены, опускаемые при эмпирической формулировке уравнений, показать, что наилучшей лебеговой переменной является мера множеств (есть и другие варианты), распространить метод на неоднородное и/или гетерогенное тело. Точность и эффективность «метода лебеговского осреднения» при решении задач переноса теплового излучения в плазме и в атмосфере Земли исследована в [19]-[21]. Оказалось, что, в сравнении с прямым решением уравнения (1), метод сокращает число требуемых арифметических операций и объем «хранимых» данных в $10^{3}-10^{4}$ раз при сохранении того же уровня точности. Правда, при использовании метода теряется информация о спектре излучения. Недостаток устранен в методе спектральных моментов.

Вторая идея состоит в использовании метода моментов - разложения спектра в ряд по системе базисных функций для нахождения решения 
уравнения переноса излучения и последующего восстановления спектра фотонов. Попытки разработать такой метод ранее предпринимались в работах [22]-[25]. Они оказались не очень удачными, т.к. базисные функции используемых разложений не передают многообразие и сложность реальных спектров илучения. Базисные функции метода [22], [23] суть гладкие функции энергии фотонов (многочлены, близкие к многочленам Лагерра). Учет переноса излучения в линиях предлагается проводить с помощью выделения соответствующих участков спектра и решения на них независимых кинетических уравнений. При числе линий больше десятка эффективность метода становится ниже эффективности многогруппового приближения. Базисные функции метода [24] учитывают резонансы поглощения фотонов. Однако предложенное разложение - по степеням $\kappa(E)$ - выполняется только для однородного тела с постоянным, не зависящим от координат макросечением $\kappa(E)$. Другое серьезное ограничение связано с тем, что степенное разложение хорошо сходится только в оптически тонком теле $\kappa(E) L<1$, где $L$ есть размер тела (случай редко встречаемый при решении практических задач из-за больших значений макросечения в линиях поглощения). Предпринятая в [25] попытка распространить метод на сечения, зависящие от координат, сильно увеличивает размерность разложения, что вкупе с низкой скоростью сходимости делает разложение малоэффективным.

В настоящей работе выполняется разложение спектра фотонов в ряд экспонент. Объединение метода лебеговского осреднения и метода моментов: (a) позволяет восстанавливать детальное спектральное распределение фотонов, (б) вносит элемент оптимизации - оптимальную сетку по мере лебеговых множеств, что делает метод еще экономичнее.

\section{1. Модель радиационно-столкновительной плазмы}

В разделе приведены уравнения модели радиационно-столкновительной плазмы в приближении полного перераспределения энергии поглощаемых фотонов в линиях поглощения/испускания. Более подробные пояснения уравнений и обсуждение моделей с неполным перераспределением можно найти в [26]-[39].

Уравнение переноса излучения. Для расчета распределения фотонов в объеме газа, плазмы требуется найти решение линейного уравнения Больцмана:

$$
\begin{aligned}
& \frac{1}{c} \frac{\partial I}{\partial t}+\mathbf{\Omega} \frac{\partial I}{\partial \mathbf{r}}+\kappa I=\int_{4 \pi} K^{s c}\left(\mathbf{\Omega}^{\prime}\right) I\left(\mathbf{\Omega}^{\prime}\right) d \mathbf{\Omega}^{\prime}+\frac{q}{4 \pi}, \\
& \frac{1}{c} \frac{\partial}{\partial t}+\mathbf{\Omega} \frac{\partial}{\partial \mathbf{r}}=\frac{1}{c} \frac{\partial}{\partial t}+\frac{\partial}{\partial l}=\frac{d}{d l}, \quad \kappa=\kappa^{a b}+\kappa^{s c}, \quad \kappa^{s c}=\int_{4 \pi} K^{s c}\left(\boldsymbol{\Omega} \mathbf{\Omega}^{\prime}\right) d \mathbf{\Omega} .
\end{aligned}
$$

Здесь $I(E, \boldsymbol{\Omega}, \mathbf{r}, t)$ - интенсивность излучения (функция распределения фотонов), зависящая от координат $(\mathbf{r}, t)$, энергии фотонов $E=h v$ и 
направления полета пучка фотонов $\boldsymbol{\Omega},(|\boldsymbol{\Omega}|=1)$. Интенсивность излучения нормируется так, что ее угловые моменты нулевого и первого порядков

$$
\int_{4 \pi} I(E, \boldsymbol{\Omega}, \mathbf{r}, t) d \boldsymbol{\Omega}=c U(E, \mathbf{r}, t), \quad \int_{4 \pi} \boldsymbol{\Omega} I(E, \boldsymbol{\Omega}, \mathbf{r}, t) d \mathbf{\Omega}=\mathbf{W}(E, \mathbf{r}, t)
$$

равны соответственно объемной спектральной плотности энергии излучения $U(E, \mathbf{r}, t)$, умноженной на скорость света «c», и потоку энергии излучения $\mathbf{W}(E, \mathbf{r}, t)$ в единичном интервале спектра. Дифференциальный оператор в уравнении (1) может быть частично свернут в производную $\partial / \partial l$ по направлению полета $\boldsymbol{\Omega}$ и полностью свернут в производную $d / d l$ вдоль траектории движения фотонов (см. Рис. 2). Траектория является характеристикой оператора и задается уравнениями движения: $\mathbf{r}(l)=\mathbf{r}_{0}+\boldsymbol{\Omega} l$, $t(l)=t_{0}+l / c$, где $l-$ характеристическая координата (путь вдоль траектории).

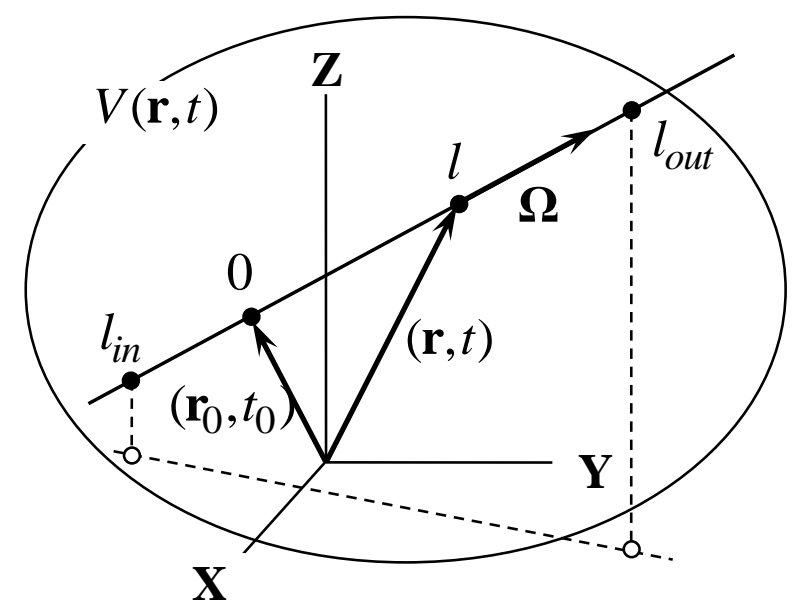

Рис. 2. Траектория пучка фотонов (характеристика). Точки входа и выхода траектории из тела имеют характеристические координаты $l_{\text {in }} u l_{\text {оut }}$.

Макросечение экстинкции фотонов $\kappa(E, \mathbf{r}, t)$ задает скорость выбытия фотонов из пучка и имеет размерность обратной длины. Оно равно сумме макросечения поглощения излучения атомами, ионами, молекулами $\kappa^{a b}(E, \mathbf{r}, t)$ и макросечения рассеяния излучения на электронах и частицах вещества в конденсированном состоянии (пыли, туманы) $\kappa^{s c}(E, \mathbf{r}, t)$. Интегральный оператор задает скорость прихода фотонов в пучок из других пучков в актах рассеяния, $\mathrm{K}^{s c}(E, \eta, \mathbf{r}, t)$ - дифференциальное сечение рассеяния, $\eta=\mathbf{\Omega}^{\prime}$ косинус угла рассеяния. Величина $q(E, \mathbf{r}, t)$ есть мощность тепловых источников излучения.

Граничные условия. На функцию распределения фотонов, движущихся от границы вглубь тела $\mathbf{\Omega n}<0$, накладывается граничное условие: 


$$
\left.I\left(\boldsymbol{\Omega}, \mathbf{r}_{i n}\right)\right|_{\mathbf{n} \mathbf{\Omega}<0}=\int_{\mathbf{n} \mathbf{\Omega}^{\prime}>0} \Sigma\left(\boldsymbol{\Omega}^{\prime} \rightarrow \boldsymbol{\Omega}, \mathbf{r}_{i n}\right) I\left(\boldsymbol{\Omega}^{\prime}\right) d \boldsymbol{\Omega}^{\prime}+\left.I^{e x t}\left(\boldsymbol{\Omega}, \mathbf{r}_{i n}\right)\right|_{\mathbf{n} \mathbf{\Omega}<0},
$$

где $\mathbf{n}$ есть вектор внешней нормали к границе, $I^{e x t}\left(E, \boldsymbol{\Omega}, \mathbf{r}_{i n}, t\right)-$ распределение «внешних» фотонов, прошедших сквозь границу или испущенных границей вглубь тела, $\Sigma\left(E, \boldsymbol{\Omega}^{\prime} \rightarrow \boldsymbol{\Omega}, \mathbf{r}_{i n}, t\right)$ - сечение отражения от границы тела.

\section{Макросечение поглощения и мощность источников излучения.} Зависимость макросечения поглощения $\kappa^{a b}(E)$ и мощности источников излучения $q(E)$ от энергии фотонов содержит множество резонансов (линий) и ступенек (границ фотоионизации), соответствующих радиационным переходам ионов, атомов, молекул между квантовыми состояниями внутренней энергии.

Для того чтобы не загромождать изложение деталями, не имеющими прямого отношения к существу метода спектральных моментов, ограничимся рассмотрением атомарной радиационно-столкновительной плазмы (без молекул и молекулярных ионов). Состояние внутренней энергии атома или иона (далее для краткости - «иона») обозначаем парой чисел $A, n$, где $A-$ атомный вес, $n$ - порядковый номер уровня внутренней энергии $E_{n}^{A}$. Состояние $A, n$ может включать несколько квантовых состояний, имеющих одинаковую (близкую) энергию $E_{n}^{A}$ и характеризуемых набором чисел. В набор $n \equiv\{Z, n, l, J\}$ может входить заряд иона $Z$, главное квантовое число $n$, орбитальное число $l$, полный вращательный момент электронов $J$. Концентрация ионов в состоянии $A, n$ обозначается символом $N_{n}^{A}$, заряд иона $Z_{n}^{A}$, статистический вес $-g_{n}^{A}$.

Будем считать, что для столкновительной плазмы применимо одножидкостное двухтемпературное приближение [40]-[43]. Распределение свободных электронов и ионов по энергии хаотических поступательных движений задается распределением Максвелла с температурами $T_{e}(\mathbf{r}, t)$ и $T_{a}(\mathbf{r}, t)$ соответственно; распределение концентраций ионов $N_{n}^{A}$ по состояниям внутренней энергии $E_{n}^{A}$ в общем случае может быть неравновесным; выполняется условие квазинейтральности, выражающее отсутствие разделения зарядов в физически бесконечно малом объеме плазмы:

$$
N_{e}=\sum_{A, n} Z_{n}^{A} N_{n}^{A}=\sum_{A} Z_{A} N_{A}=\langle Z\rangle N_{a}=\langle Z\rangle \frac{N_{A v} \rho}{\langle A\rangle} .
$$

Здесь $N_{e}$ и $N_{a}$ - концентрация электронов и ионов, $\langle Z\rangle$ и $\langle A\rangle-$ средний заряд и средний атомный вес ионов, $\rho$ - плотность, $N_{A v}$ - число Авогадро. 
Макросечение поглощения фотонов $\kappa^{a b}$ может быть представлено в виде суммы макросечений поглощения энергии ионами $\kappa^{r \rightarrow b}$ и электронами $\kappa^{r \rightarrow e}$ :

$$
\begin{gathered}
\kappa^{a b}=\sum_{A, n} N_{n}^{A}\left[1-e^{-E / T_{e}}\right]\left[\sum_{m>n}\left[1+\frac{\left.1-N_{m}^{A} \bar{N}_{n}^{A} / N_{n}^{A} \bar{N}_{m}^{A}\right]}{e^{E / T_{e}}-1}\right] \sigma_{n \rightarrow m}^{A}+N_{e} \sigma_{n, e \rightarrow e}^{A}\right], \\
\kappa^{r \rightarrow b}=\sum_{A, n} N_{n}^{A}\left[1-e^{-E / T_{e}}\right] \sum_{m>n}\left[1+\frac{\left.1-N_{m}^{A} \bar{N}_{n}^{A} / N_{n}^{A} \bar{N}_{m}^{A}\right]}{e^{E / T_{e}}-1}\right] \sigma_{n \rightarrow m}^{A, r \rightarrow b}, \\
\kappa^{r \rightarrow e}=\sum_{A, n} N_{n}^{A}\left[1-e^{-E / T_{e}}\right]\left[\sum_{m>n}\left[1+\frac{\left.1-N_{m}^{A} \bar{N}_{n}^{A} / N_{n}^{A} \bar{N}_{m}^{A}\right]}{e^{E / T_{e}}-1}\right] \sigma_{n \rightarrow m}^{A, r \rightarrow e}+N_{e} \sigma_{n, e \rightarrow e}^{A}\right], \\
\kappa^{a b}(E, \mathbf{r}, t)=\kappa^{r \rightarrow b}(E, \mathbf{r}, t)+\kappa^{r \rightarrow e}(E, \mathbf{r}, t), \quad \sigma_{n \rightarrow m}^{A}=\sigma_{n \rightarrow m}^{A, r \rightarrow b}+\sigma_{n \rightarrow m}^{A, r \rightarrow e}, \\
\sigma_{n \rightarrow m}^{A, r \rightarrow b}=\frac{E_{m}^{A}-E_{n}^{A}}{E} \sigma_{n \rightarrow m}^{A}, \quad \sigma_{n \rightarrow m}^{A, r \rightarrow e}=\left[1-\frac{E_{m}^{A}-E_{n}^{A}}{E}\right] \sigma_{n \rightarrow m}^{A} .
\end{gathered}
$$

Сечение $\sigma_{n \rightarrow m}^{A}$ описывает суммарную скорость переходов иона вверх по шкале энергий из состояния с энергией $E_{n}^{A}$ в состояние с большей энергией $E_{m}^{A}$, сопровождаемых поглощением фотона. В атомарной плазме это процессы радиационного возбуждения и фотоионизации. $\sigma_{n, e \rightarrow e}^{A}$ есть сечение тормозного поглощения фотона (столкновения свободного электрона с ионом, в результате которого электрон увеличивает энергию на энергию фотона, состояние иона не меняется). Сечения процессов индуцированного испускания фотонов присоединены к сечениям переходов вверх со знаком минус. Сечение $\sigma_{n \rightarrow m}^{A, r \rightarrow b}$ описывает долю энергии фотона, затрачиваемую на возбуждение иона, сечение $\sigma_{n \rightarrow m}^{A, r \rightarrow e}$ - долю энергии, передаваемую свободному электрону в акте фотоионизации. В формуле для макросечения $\kappa^{r \rightarrow e}$ суммирование выполняется по всем переходам, включая процессы радиационного возбуждения, в которых электрон не участвует. Эти слагаемые автоматически выпадают из суммы из-за резонансного характера сечения $\sigma_{n \rightarrow m}^{A}(E)$ на энергии перехода $E \approx E_{m}^{A}-E_{n}^{A}$.

Мощность источников излучения $q(E, \mathbf{r}, t)$ в приближении полного перераспределения энергии поглощаемых фотонов может быть представлена в виде произведения макросечения испускания фотонов $\kappa^{e m}(E, \mathbf{r}, t)$ и планковского распределения $U^{P l}\left(E, T_{e}\right)$ при температуре электронов $T_{e}(\mathbf{r}, t)$ :

$$
q(E, \mathbf{r}, t)=c \kappa^{e m} U^{P l}\left(E, T_{e}\right),
$$




$$
\begin{aligned}
& \kappa^{e m}(E, \mathbf{r}, t)=\left[1-e^{-E / T_{e}}\right] \sum_{A, n}\left[\sum_{m>n} N_{m}^{A} \sigma_{m \rightarrow n}^{A}+N_{n}^{A} N_{e} \sigma_{n, e \rightarrow e}^{A}\right]= \\
& =\left[1-e^{-E / T_{e}}\right] \sum_{A, n}\left[\sum_{m>n} N_{m}^{A} \frac{\bar{N}_{n}^{A}}{\bar{N}_{m}^{A}} \sigma_{n \rightarrow m}^{A}+N_{n}^{A} N_{e} \sigma_{n, e \rightarrow e}^{A}\right], \\
& U^{P l}\left(E, T_{e}\right)=\frac{4 \sigma}{c} \frac{15}{\pi^{4}} \frac{E^{3}}{e^{E / T_{e}}-1}, \quad U^{P l}\left(T_{e}\right)=\int U^{P l}\left(E, T_{e}\right) d E=\frac{4 \sigma T_{e}^{4}}{c}, \\
& \sigma=\frac{2 \pi^{5} Y^{4}}{15 c^{2} h^{3}}=1.028301 \cdot 10^{12} \frac{\ni р г}{c e \kappa \cdot c M^{2}{ } B^{4}}, \quad Y=1.602176565 \cdot 10^{-12} \frac{\ni p 2}{{ }^{\prime} B} \text {. }
\end{aligned}
$$

Сечение $\sigma_{m \rightarrow n}^{A}$ описывает суммарную скорость переходов иона вниз по шкале энергий, сопровождаемых испусканием фотона. В атомарной плазме это радиационный распад возбужденного состояния и фоторекомбинация. $\sigma$ постоянная Стефана-Больцмана, $Y$ - масштабный множитель перевода единиц измерения энергии из эВ в эрг.

Сечения испускания фотонов, как и сечения поглощения, можно представить в виде суммы сечений излучения энергии ионами и электронами:

$$
\begin{aligned}
& \kappa^{e m}=\kappa^{b \rightarrow r}+\kappa^{e \rightarrow r}, \quad \sigma_{m \rightarrow n}^{A}=\sigma_{m \rightarrow n}^{A, b \rightarrow r}+\sigma_{m \rightarrow n}^{A, e \rightarrow r}, \\
& \kappa^{b \rightarrow r}=\left[1-e^{-E / T_{e}}\right] \sum_{A, n} \sum_{m>n} N_{m}^{A} \sigma_{m \rightarrow n}^{A, b \rightarrow r}=\left[1-e^{-E / T_{e}}\right] \sum_{A, n} \sum_{m>n} N_{m}^{A} \frac{\bar{N}_{n}^{A}}{\bar{N}_{m}^{A}} \sigma_{n \rightarrow m}^{A, r \rightarrow b}, \\
& \kappa^{e \rightarrow r}=\left[1-e^{-E / T_{e}}\right] \sum_{A, n}\left[\sum_{m>n} N_{m}^{A} \sigma_{m \rightarrow n}^{A, e \rightarrow r}+N_{n}^{A} N_{e} \sigma_{n, e \rightarrow e}^{A}\right], \\
& \sigma_{m \rightarrow n}^{A, b \rightarrow r}=\frac{E_{m}^{A}-E_{n}^{A}}{E} \sigma_{m \rightarrow n}^{A}=\frac{\bar{N}_{n}^{A}}{\bar{N}_{m}^{A}} \sigma_{n \rightarrow m}^{A, r \rightarrow b}, \\
& \sigma_{m \rightarrow n}^{A, e \rightarrow r}=\left[1-\frac{E_{m}^{A}-E_{n}^{A}}{E}\right] \sigma_{m \rightarrow n}^{A}=\frac{\bar{N}_{n}^{A}}{\bar{N}_{m}^{A}} \sigma_{n \rightarrow m}^{A, r \rightarrow e} .
\end{aligned}
$$

Сечения переходов вверх и переходов вниз выражаются друг через друга с помощью соотношений детального баланса:

$$
\bar{N}_{n}^{A} \sigma_{n \rightarrow m}^{A}=\bar{N}_{m}^{A} \sigma_{m \rightarrow n}^{A} .
$$

Верхней чертой в (5)-(8) обозначены равновесные концентрации ионов, удовлетворяющие соотношениям ионизационного равновесия Саха-Больцмана: 


$$
\frac{\bar{N}_{m}^{A}}{g_{m}^{A}}=\frac{\bar{N}_{n}^{A}}{g_{n}^{A}} \cdot\left[\frac{g_{e}}{N_{e}}\right]^{Z_{m}^{A}-Z_{n}^{A}} \exp \left[-\frac{E_{m}^{A}-E_{n}^{A}}{T_{e}}\right], \quad g_{e}\left(T_{e}\right)=\left[\frac{[32]^{1 / 3} \pi m_{e} Y T_{e}}{h^{2}}\right]^{\frac{3}{2}} .
$$

Равновесные концентрации возбужденных состояний ионов и планковское распределение введены в формулы (5), (6) для компактности записи. Формулы справедливы при неравновесном распределении ионов по состояниям.

Если вещество находится в локальном термодинамическом равновесии (ЛТР), то соотношений ионизационного равновесия (9), условия квазинейтральности плазмы (4) и нормировочных соотношений

$$
N_{A}(\mathbf{r}, t)=\sum N_{n}^{A}
$$

достаточно, чтобы рассчитать равновесное распределение ионов $\bar{N}_{n}^{A}$, равновесные макросечения поглощения, испускания и мощность тепловых источников. В ЛТР они связаны простыми соотношениями:

$$
\kappa_{L T E}^{a b}=\kappa_{L T E}^{e m}, \quad q_{L T E}=c \kappa_{L T E}^{a b} U^{P l}\left(E, T_{e}\right)=\kappa_{L T E}^{a b} 4 \sigma \frac{15}{\pi^{4}} \frac{E^{3}}{e^{E / T_{e}}-1} .
$$

Уравнения поуровневой кинетики. В отсутствие термодинамического равновесия концентрации ионов $N_{n}^{A}$ в состояниях $A, n$ находятся из совместного решения уравнения переноса излучения (1) и уравнений радиационно-столкновительной поуровневой кинетики:

$$
\begin{gathered}
N_{A} \frac{d}{d t}\left[\frac{N_{n}^{A}}{N_{A}}\right]=-\sum_{m>n} C_{n \rightarrow m}^{A}\left[N_{n}^{A}-N_{m}^{A} \frac{\bar{N}_{n}^{A}}{\bar{N}_{m}^{A}}\right]+\sum_{m<n} C_{m \rightarrow n}^{A}\left[N_{m}^{A}-N_{n}^{A} \frac{\bar{N}_{m}^{A}}{\bar{N}_{n}^{A}}\right]- \\
-\frac{c}{Y} \sum_{m>n} \int_{0}^{\infty} \sigma_{n \rightarrow m}^{A}\left[1-e^{-E / T_{e}}\right]\left[N _ { n } ^ { A } U \left[1+\frac{\left.\left.1-N_{m}^{A} \bar{N}_{n}^{A} / N_{n}^{A} \bar{N}_{m}^{A}\right]-N_{m}^{A} \frac{\bar{N}_{n}^{A}}{e^{E / T_{e}}-1} U^{P l}\right] \frac{d E}{E}-}{+\frac{c}{Y} \sum_{m<n} \int_{0}^{\infty} \sigma_{m \rightarrow n}^{A}\left[1-e^{-E / T_{e}}\right]\left[N_{m}^{A} U\left[1+\frac{1-N_{n}^{A} \bar{N}_{m}^{A} / N_{m}^{A} \bar{N}_{n}^{A}}{e^{E / T_{e}}-1}\right]-N_{n}^{A} \frac{\bar{N}_{m}^{A}}{\bar{N}_{n}^{A}} U^{P l}\right] \frac{d E}{E},}\right.\right. \\
\frac{d}{d t}=\frac{\partial}{\partial t}+\mathbf{u} \frac{\partial}{\partial \mathbf{r}}, \quad \bar{N}_{n}^{A} C_{n \rightarrow m}^{A}=\bar{N}_{m}^{A} C_{m \rightarrow n}^{A} .
\end{gathered}
$$

Здесь $C_{n \rightarrow m}^{A}$ - скорость безызлучательных переходов из состояния с энергией $E_{n}^{A}$ в состояние с большей энергией $E_{m}^{A}$ при столкновениях с электронами (возбуждения иона, ионизации и автоионизации). Скорости обратных переходов вниз по шкале энергий (тушения возбужденного состояния, трехчастичной рекомбинации и диэлектронного захвата) выражены через скорости переходов вверх с помощью соотношений детального баланса. $d / d t-$ 
субстанциональная производная (substance (англ.) - вещество), $\mathbf{u}$ - скорость. При записи уравнений мы пренебрегли процессами атомарной диффузии.

Решение системы уравнений поуровневой кинетики связано с решением уранения переноса излучения через плотность энергии излучения $U$.

Обзоры методов расчета сечений столкновительных и радиационных переходов ионов в атомарной плазме и описание процедур осреднения сечений по электронным конфигурациям можно найти в [44]-[53].

Агрегирование спектра энергий ионов. В целях уменьшения размерности задачи по дискретному спектру внутренней энергии ионов выполним объединение (агрегирование) состояний с близкими энергиями в группы состояний, или группы уровней энергии. Прием широко используется при решении кинетических задач (см., например, [26], [30], [39], [54], [55]).

В атомарной плазме одного элемента, в которой присутствуют ионы разных зарядов $Z, Z_{\min } \leq Z \leq Z_{\max }$ числом $\Delta Z=Z_{\max }-Z_{\min }, \Delta Z=0,1,2, \ldots$, вводится как минимум $K_{\min }=\Delta Z+1$ групп состояний. Группа состоит из возбужденных состояний иона с зарядом «Z-1» и основного состояния иона с зарядом «Z». (Иногда в группу добавляется первое, второе возбужденные состояния иона «Z»). При таком объединении каждый из ионов описывается «двухуровневой моделью». С увеличением числа групп точность описания спектра энергий ионов увеличивается. Если для иона использовать $M$ групп состояний $(M+1$-уровневую модель), то общее число групп равно $K=M[\Delta Z+1] H$, где $H-$ число элементов плазмы.

При объединении состояний для простоты будем считать, что концентрации ионов в состояниях $A, n$, входящих в группу $A, k, \quad n \in(k)$ распределены в соответствии с соотношениями Саха-Больцмана (9) с температурой электронов $T_{e}$. (Возможен вариант объединения с эффективной температурой группы уровней). Соотношения преобразуются к виду:

$$
\begin{gathered}
\frac{N_{n}^{A}}{g_{n}^{A}}=\frac{N_{k}^{A}}{g_{k}^{A}} \cdot\left[\frac{g_{e}}{N_{e}}\right]^{Z_{n}^{A}-Z_{k}^{A}} \exp \left[-\frac{E_{n}^{A}-E_{k}^{A}}{T_{e}}\right], \quad g_{e}\left(T_{e}\right)=\left[\frac{[32]^{1 / 3} \pi m_{e}}{h^{2}} Y T_{e}\right]^{\frac{3}{2}}, \\
N_{k}^{A}=\sum_{n \in(k)} N_{n}^{A}, \quad g_{k}^{A}=\sum_{n \in(k)} g_{n}^{A}\left[\frac{g_{e}}{N_{e}}\right]^{Z_{n}^{A}-Z_{k}^{A}} \exp \left[-\frac{E_{n}^{A}-E_{k}^{A}}{T_{e}}\right] .
\end{gathered}
$$

Здесь $N_{k}^{A}$ - концентрация ионов в группе состояний $A, k, g_{k}^{A}\left(T_{e}, N_{e}\right)$ статистический вес группы состояний. Энергия $E_{k}^{A}\left(T_{e}, N_{e}\right)$ и средний заряд $Z_{k}^{A}\left(T_{e}, N_{e}\right)$ группы состояний $A, k$ суть функции параметров $\left(T_{e}, N_{e}\right)$ и находятся подстановкой соотношений (13) в уравнения: 


$$
E_{k}^{A}=\sum_{n \in(k)} E_{n}^{A} N_{n}^{A} / \sum_{n \in(k)} N_{n}^{A}, \quad Z_{k}^{A}=\sum_{n \in(k)} Z_{n}^{A} N_{n}^{A} / \sum_{n \in(k)} N_{n}^{A} .
$$

Расчет концентраций ионов $N_{k}^{A}$ в группах состояний может выполняться в двух вариантах. В одном варианте используется агрегированная система уравнений кинетики, получаемая непосредственным суммированием уравнений детальной системы (12) по группам состояний. В альтернативном варианте используется система для групп внутренней энергии ионов $\left[E_{k}^{A} N_{k}^{A}\right]=\rho \varepsilon_{k}^{A} / Y$. Концентрации ионов $N_{k}^{A}$ вычисляются опосредованно после нахождения энергии группы $\left[E_{k}^{A} N_{k}^{A}\right]$, делением произведения на энергию $E_{k}^{A}$. В данной работе мы придерживаемся второго подхода. На наш взгляд он более органично вписывается в общую структуру уравнений радиационно-столкновительной модели плазмы (1)-(29). Для вывода агрегированной системы кинетических уравнений сначала умножим каждое из уравнений детальной системы (12) на энергию $E_{n}^{A}$, а затем выполним суммирование по группам уровней:

$$
\begin{aligned}
N_{A} \frac{d}{d t}\left[\frac{E_{k}^{A} N_{k}^{A}}{N_{A}}\right]=\sum_{l>k}\left[C_{k \rightarrow l}^{A, e \rightarrow b} \frac{E_{l}^{A}-E_{k}^{A}}{2}-C_{k \rightarrow l}^{A+} \frac{E_{l}^{A}+E_{k}^{A}}{2}\right]\left[N_{k}^{A}-N_{l}^{A} \frac{\bar{N}_{k}^{A}}{\bar{N}_{l}^{A}}\right]+ \\
+\sum_{l<k}\left[C_{l \rightarrow k}^{A, e \rightarrow b} \frac{E_{k}^{A}-E_{l}^{A}}{2}+C_{l \rightarrow k}^{A+} \frac{E_{l}^{A}+E_{k}^{A}}{2}\right]\left[N_{l}^{A}-N_{k}^{A} \frac{\bar{N}_{l}^{A}}{\bar{N}_{k}^{A}}\right]+ \\
\left.+\frac{c}{Y} \sum_{l \geq k} \int_{0}^{\infty} \frac{\sigma_{k \rightarrow l}^{A, r \rightarrow b}-\sigma_{k \rightarrow l}^{A+}\left[N_{k}^{A} U\left[1+\frac{1-N_{l}^{A} \bar{N}_{k}^{A} / N_{k}^{A} \bar{N}_{l}^{A}}{2}\right]-N_{l}^{A / T_{e}}-1\right.}{2} \frac{\bar{N}_{k}^{A}}{\bar{N}_{l}^{A}} U^{P l}\right] d E+ \\
\left.+\frac{c}{Y} \sum_{l \leq k}^{\infty} \int_{0}^{\infty} \frac{\sigma_{l \rightarrow k}^{A, r \rightarrow b}+\sigma_{l \rightarrow k}^{A+}\left[N_{l}^{A} U\left[1+\frac{1-N_{k}^{A} \bar{N}_{l}^{A} / N_{l}^{A} \bar{N}_{k}^{A}}{2}\right]-N_{k}^{A / T_{e}}-1\right.}{2} \frac{\bar{N}_{l}^{A}}{\bar{N}_{k}^{A}} U^{P l}\right] d E .
\end{aligned}
$$

Сечения переходов между группами состояний вычисляются суммированием детальных сечений по конечным состояниям переходов и осреднением по начальным состояниям:

$$
\begin{aligned}
& C_{k \rightarrow l}^{A+}\left(T_{e}, N_{e}\right)=\sum_{n}^{n \in(k)} \sum_{m}^{m \in(l)} \frac{E_{m}^{A}+E_{n}^{A}}{E_{l}^{A}+E_{k}^{A}} C_{n \rightarrow m}^{A} \frac{N_{n}^{A}}{N_{k}^{A}}, \\
& C_{k \rightarrow l}^{A, e \rightarrow b}\left(T_{e}, N_{e}\right)=\sum_{n}^{n \in(k)} \sum_{m}^{m \in(l)} \frac{E_{m}^{A}-E_{n}^{A}}{E_{l}^{A}-E_{k}^{A}} C_{n \rightarrow m}^{A} \frac{N_{n}^{A}}{N_{k}^{A}}, \quad(l>k), \quad C_{k \rightarrow k}^{A, e \rightarrow b}=0, \\
& \sigma_{k \rightarrow l}^{A+}\left(E, T_{e}, N_{e}\right)=\left[1-e^{-E / T_{e}}\right] \sum_{n}^{n \in(k)} \sum_{m>n}^{m \in(l)} \frac{E_{m}^{A}+E_{n}^{A}}{E} \sigma_{n \rightarrow m}^{A} \frac{N_{n}^{A}}{N_{k}^{A}},
\end{aligned}
$$




$$
\begin{aligned}
\sigma_{k \rightarrow l}^{A, r \rightarrow b}\left(E, T_{e}, N_{e}\right) & =\left[1-e^{-E / T_{e}}\right] \sum_{n}^{n \in(k)} \sum_{m>n}^{m \in(l)} \frac{E_{m}^{A}-E_{n}^{A}}{E} \sigma_{n \rightarrow m}^{A} \frac{N_{n}^{A}}{N_{k}^{A}}= \\
& =\left[1-e^{-E / T_{e}}\right] \sum_{n}^{n \in(k)} \sum_{m>n}^{m \in(l)} \sigma_{n \rightarrow m}^{A, r \rightarrow b} \frac{N_{n}^{A}}{N_{k}^{A}}, \quad(l \geq k) .
\end{aligned}
$$

Сечения $C_{k \rightarrow l}^{A+}$ и $\sigma_{k \rightarrow l}^{A+}$ задают скорости процессов перераспределения энергии между состояниями $A, k$. Сечения $C_{k \rightarrow l}^{A, e \rightarrow b}$ и $\sigma_{k \rightarrow l}^{A, r \rightarrow b}$ - скорости прихода энергии в состояние $A, k$ соответственно от свободных электронов и от излучения. Сечения радиационных переходов для удобства домножены на множитель $\left[1-e^{-E / T_{e}}\right]$. Обращаем внимание, что уравнения (14) учитывают излучательные процессы обмена энергией внутри группы состояний $A, k \rightarrow A, k,(l=k)$. Соответствующие сечения радиационных переходов не равны нулю. Если сложить уравнения (14), то мы получим уравнение баланса внутренней энергии ионов (24) (с точностью до масштабного множителя $Y$ перевода единиц измерения из эВ в эрг).

Система (14) состоит из $K \approx M[\Delta Z+1] H$ уравнений и содержит примерно $[M+1]^{2}[\Delta Z+1] H$ сечений переходов. В детальной системе (12) число сечений равно $R[R+1][\Delta+1] H / 2$, где $R$ - число учитываемых уровней иона. Одно сечение радиационных переходов между группами состояний $\sigma_{k \rightarrow l}^{A}$ содержит в среднем $R^{2} /[M+1]^{2}$ линий (или больше, если учитывается тонкая структура мультиплета). При объединении состояний ионов в группы мы не производим объединения контуров отдельных линий, принадлежащих тем или иным переходам, в один «размазанный» контур. Метод спектральных моментов позволяет сохранить многообразие линий с их исходными контурами.

Также введем сечение $\sigma_{k \rightarrow l}^{A, r \rightarrow e}$ прихода энергии от излучения к свободным электронам, дополняющее сечение $\sigma_{k \rightarrow l}^{A, r \rightarrow b}$ до полного сечения $\sigma_{k \rightarrow l}^{A}$, и сечение прихода энергии излучения к свободным электронам в тормозных процессах:

$$
\begin{aligned}
& \sigma_{k \rightarrow l}^{A, r \rightarrow e}\left(E, T_{e}, N_{e}\right)=\left[1-e^{-E / T_{e}}\right] \sum_{n}^{n \in(k)} \sum_{m>n}^{m \in(l)}\left[1-\frac{E_{m}^{A}-E_{n}^{A}}{E}\right] \sigma_{n \rightarrow m}^{A} \frac{N_{n}^{A}}{N_{k}^{A}}, \\
& \sigma_{k \rightarrow l}^{A}\left(E, T_{e}, N_{e}\right)=\sigma_{k \rightarrow l}^{A, r \rightarrow b}+\sigma_{k \rightarrow l}^{A, r \rightarrow e}=\left[1-e^{-E / T_{e}}\right] \sum_{n}^{n \in(k)} \sum_{m>n}^{m \in(l)} \sigma_{n \rightarrow m}^{A} \frac{N_{n}^{A}}{N_{k}^{A}}, \\
& \sigma_{k, e \rightarrow e}^{A}\left(E, T_{e}, N_{e}\right)=\left[1-e^{-E / T_{e}}\right] \sum_{n}^{n \in(k)} \sigma_{n, e \rightarrow e}^{A} \frac{N_{n}^{A}}{N_{k}^{A}} .
\end{aligned}
$$


Формулы (4)-(8) для расчета концентрации свободных электронов, макросечений поглощения и испускания излучения при объединении состояний сохраняют свой функциональный вид. Достаточно заменить индекс $n$ на $k$ и условие суммирования $m>n$ на условие $l \geq k$. Приведем, к примеру, выражение для макросечения поглощения (5):

$\kappa^{a b}=\sum_{A, k} \kappa_{A, k}, \quad \kappa_{A, k}=N_{k}^{A}\left[\sum_{l \geq k} \sigma_{k \rightarrow l}^{A}\left[1+\frac{1-N_{l}^{A} \bar{N}_{k}^{A} / N_{k}^{A} \bar{N}_{l}^{A}}{e^{E / T_{e}}-1}\right]+N_{e} \sigma_{k, e \rightarrow e}^{A}\right]$.

Уравнения динамики плазмы. Радиационно-столкновительные процессы в плазме, описываемые кинетическими уравнениями (1)-(12), обычно протекают на фоне движения вещества и интенсивного обмена энергией между компонентами плазмы. Приведем газодинамические уравнения, дополняющие радиационно-столкновительную модель плазмы.

Уравнение неразрывности:

$$
\frac{\partial \rho}{\partial t}+\frac{\partial\left[\rho u_{i}\right]}{\partial r_{i}}=0, \text { или } \rho \frac{d}{d t}\left[\frac{1}{\rho}\right]=\frac{\partial u_{i}}{\partial r_{i}}, \quad \rho_{\left[g \cdot \mathrm{cm}^{-3}\right]}=\frac{\sum A N_{A}}{N_{A v}}=\frac{\langle A\rangle N_{a}}{N_{A v}} .
$$

Уравнение движения:

$$
\frac{\partial\left[\rho u_{i}\right]}{\partial t}+\frac{\partial}{\partial r_{j}}\left[\rho u_{i} u_{j}+P_{a} \delta_{i j}-\sigma_{i j}^{a}+P_{e} \delta_{i j}-\sigma_{i j}^{e}+\underline{P_{i j}^{r}}\right]=F_{i},
$$

или $\rho \frac{d u_{i}}{d t}+\frac{\partial}{\partial r_{j}}\left[P_{a} \delta_{i j}-\sigma_{i j}^{a}+P_{e} \delta_{i j}-\sigma_{i j}^{e}+\underline{P_{i j}^{r}}\right]=F_{i}$.

$\mathbf{F}$ - внешняя сила. Уравнение движения дополняется соотношением для расчета тензора давления излучения $P_{i j}^{r}$ (см. (25)) и уравнениями состояния для расчета тензоров вязкости $\sigma_{i j}^{a}$, $\sigma_{i j}^{e}$, давления ионов $P_{a}$ и давления электронов $P_{e}$ :

$$
P_{a}=P_{a}\left(T_{a}, \rho\right) \approx \frac{Y N_{A v} \cdot \rho}{\langle A\rangle} \cdot T_{a}, \quad P_{e}=P_{e}\left(T_{e}, \rho\right) \approx \frac{Y N_{A v} \rho}{\langle A\rangle}\langle Z\rangle T_{e}
$$

В неидеальной плазме давление задается в виде табличных функций от термодинамических параметров. В разреженной плазме можно пользоваться формулами для идеальной плазмы, указанными знаком приближенного равенства.

Уравнение баланса полной энергии вещества и излучения:

$$
\frac{\partial}{\partial t}\left[\frac{\rho u^{2}}{2}+\rho \varepsilon_{a}+\rho \varepsilon_{e}+\rho \varepsilon_{b}+\underline{U}\right]+\frac{\partial}{\partial r_{i}}\left[\frac{\rho u^{2}}{2}+\rho \varepsilon_{a}+\rho \varepsilon_{e}+\rho \varepsilon_{b}+\underline{U}\right] u_{i}+
$$




$$
+\frac{\partial}{\partial r_{i}}\left[W_{i}^{a}+W_{i}^{e}+W_{i}\right]+\frac{\partial}{\partial r_{i}}\left[P_{a} \delta_{i j}-\sigma_{i j}^{a}+P_{e} \delta_{i j}-\sigma_{i j}^{e}+\underline{P_{i j}^{r}}\right] u_{j}=F_{i} u_{i}+Q_{a}+Q_{e},
$$

или $\rho \frac{d}{d t}\left[\varepsilon_{a}+\varepsilon_{e}+\varepsilon_{b}+\frac{U}{\underline{\rho}}\right]+\frac{\partial}{\partial r_{i}}\left[W_{i}^{a}+W_{i}^{e}+W_{i}\right]+$

$$
+\left[P_{a} \delta_{i j}-\sigma_{i j}^{a}+P_{e} \delta_{i j}-\sigma_{i j}^{e}+\underline{P_{i j}^{r}}\right] \frac{\partial u_{j}}{\partial r_{i}}=Q_{a}+Q_{e} .
$$

Все виды энергии (см. Таблица 1), кроме внутренней энергии ионов и энергии поля излучения, суть функции термодинамических параметров.

Таблица 1. Виды энергии в атомарной излучающей столкновительной плазме

\begin{tabular}{|c|l|c|l|}
\hline & Виды энергии & Обозначение & Состояние \\
\hline 1 & $\begin{array}{l}\text { Гидродинамическая (механическая) } \\
\text { Энергия движения вещества }\end{array}$ & $\rho u^{2} / 2$ & \\
\hline 2 & $\begin{array}{l}\text { Энергия хаотических поступательных } \\
\text { движений ионов (атомов и ионов) }\end{array}$ & $\rho \varepsilon_{a}\left(T_{a}, \rho\right)$ & равновесная \\
\hline 3 & $\begin{array}{l}\text { Энергия хаотических движений } \\
\text { свободных электронов }\end{array}$ & $\rho \varepsilon_{e}\left(T_{e}, \rho\right)$ & равновесная \\
\hline 4 & Внутренняя энергия ионов & $\rho \varepsilon_{b}$ & $\begin{array}{l}\text { равновесная или } \\
\text { неравновесная }\end{array}$ \\
\hline 5 & Энергия поля излучения & $U$ & неравновесная \\
\hline
\end{tabular}

Уравнения баланса энергии $\varepsilon_{a}$ хаотического движения ионов:

$$
\begin{gathered}
\rho \frac{d \varepsilon_{a}}{d t}+\frac{\partial W_{i}^{a}}{\partial r_{i}}+\left[P_{a} \delta_{i j}-\sigma_{i j}^{a}\right] \frac{\partial u_{j}}{\partial r_{i}}=Q^{e \rightarrow a}+Q_{a}, \\
W_{i}^{a}=-\chi_{a} \frac{\partial T_{a}}{\partial r_{i}}, \quad \varepsilon_{a}\left(T_{a}, \rho\right) \approx \frac{3 Y N_{A v}}{2\langle A\rangle} T_{a} .
\end{gathered}
$$

$Q_{a}$ - внешний источник тепловой энергии ионов, $\mathbf{W}^{a}$ и $\chi_{a}-$ поток и коэффициент ионной теплопроводности.

Уравнения баланса энергии $\varepsilon_{e}$ свободных электронов:

$$
\begin{aligned}
& \rho \frac{d \varepsilon_{e}}{d t}+\frac{\partial W_{i}^{e}}{\partial r_{i}}+\left[P_{e} \delta_{i j}-\sigma_{i j}^{e}\right] \frac{\partial u_{j}}{\partial r_{i}}=-Q^{e \rightarrow a}-Q^{e \rightarrow b}-Q^{e \rightarrow r}+Q_{e}, \\
& W_{i}^{e}=-\chi_{e} \frac{\partial T_{e}}{\partial r_{i}}, \quad \varepsilon_{e}\left(T_{e}, \rho\right) \approx \frac{3 Y N_{A v}}{2\langle A\rangle}\langle Z\rangle T_{e} .
\end{aligned}
$$


$Q_{e}-$ внешний источник тепловой энергии электронов, $\mathbf{W}^{e}$ и $\chi_{e}-$ поток и коэффициент электронной теплопроводности.

Уравнение баланса внутренней энергии ионов $\varepsilon_{b}$ :

$$
\rho \frac{d \varepsilon_{b}}{d t}=Q^{e \rightarrow b}-Q^{b \rightarrow r}, \quad \rho \varepsilon_{b}(\mathbf{r}, t)=\sum_{A} \rho \varepsilon_{A}=Y \sum_{A, n} E_{n}^{A} N_{n}^{A} .
$$

Уравнение может быть получено из (14) или (12) суммированием энергий в дискретных состояниях спектра ионов.

Уравнения баланса энергии излучения $U$ :

$$
\begin{aligned}
& \rho \frac{d}{d t}\left[\frac{U}{\rho}\right]+\frac{P_{i j}^{r} \frac{\partial u_{j}}{\partial r_{i}}}{+}+\frac{\partial W_{i}}{\partial r_{i}}=Q^{b \rightarrow r}+Q^{e \rightarrow r}, \\
& \frac{\rho}{c \frac{d}{d t}\left[\frac{W_{i}}{\rho}\right]}+\frac{W_{j}}{c} \cdot \frac{\partial u_{i}}{\partial r_{j}}+c \frac{\partial P_{i j}^{r}}{\partial r_{j}}+\int_{0}^{\infty}\left[\kappa^{a b}+\kappa^{s c}-\kappa_{1}^{s c}\right] W_{i}(E) d E=0, \\
& U(\mathbf{r}, t)=\int_{0}^{\infty} U(E) d E=\frac{1}{c} \int_{0}^{\infty} \int_{4 \pi} I d \mathbf{\Omega} d E, \quad \mathbf{W}(\mathbf{r}, t)=\int_{0}^{\infty} \mathbf{W}(E) d E=\int_{0}^{\infty} \int_{4 \pi} \mathbf{\Omega} I d \mathbf{\Omega} d E, \\
& P_{i j}^{r}(\mathbf{r}, t)=\int_{0}^{\infty} P_{i j}^{r}(E) d E=\frac{1}{c} \int_{0}^{\infty} \int_{4 \pi} \Omega_{i} \Omega \Omega_{j} I d \mathbf{\Omega} d E, \quad \kappa_{1}^{s c}(E, \mathbf{r}, t)=2 \pi \int_{-1}^{1} \eta \mathrm{K}^{s c}(\eta) d \eta .
\end{aligned}
$$

Здесь интенсивность излучения $I$ и ее угловые моменты: плотность энергии излучения $U$, поток энергии излучения $\mathbf{W}$ и тензор давления излучения $P_{i j}^{r}-$ относятся к сопутствующей системе отсчета, движущейся вместе с жидкой частицей, $\kappa_{1}^{s c}-$ первый угловой момент дифференциального сечения рассеяния.

Обмен энергией. Скорости обмена энергией между компонентами плазмы $Q^{e \rightarrow a}, Q^{e \rightarrow b}, Q^{e \rightarrow r}, Q^{b \rightarrow r}$ входят в уравнения баланса энергии (22)-(25) дважды: один раз со знаком плюс, второй - со знаком минус.

Скорость обмена энергией хаотического движения между электронами и ионами:

$$
Q^{e \rightarrow a}=\frac{Y N_{A v} \rho}{\langle A\rangle} C^{e \rightarrow a}\left[T_{e}-T_{a}\right]
$$

Формулы для расчета кинетического коэффициента обмена $C^{e \rightarrow a}$, а также коэффициентов теплопроводности $\chi_{e}, \chi_{a}$ и тензоров вязкости $\sigma_{i j}^{e}, \sigma_{i j}^{a}$ при разных физических параметрах плазмы можно найти в [40]-[43]. 
Скорость обмена энергией между свободными электронами и внутренней энергией ионов в безызлучательных столкновениях:

$$
\begin{aligned}
Q^{e \rightarrow b} & =Y \sum_{A, k} \sum_{l>k}\left[E_{l}^{A}-E_{k}^{A}\right]\left[N_{k}^{A} C_{k \rightarrow l}^{A}-N_{l}^{A} C_{l \rightarrow k}^{A}\right]= \\
& =Y \sum_{A, k} \sum_{l>k}\left[E_{l}^{A}-E_{k}^{A}\right]\left[N_{k}^{A} \frac{\bar{N}_{l}^{A}}{\bar{N}_{k}^{A}}-N_{l}^{A}\right] C_{l \rightarrow k}^{A} .
\end{aligned}
$$

Во второй части формулы (27) скорости переходов «вверх» выражены через скорости переходов «вниз».

Скорость обмена между внутренней энергией ионов и полем излучения:

$$
Q^{b \rightarrow r}=c \int_{0}^{\infty}\left[\kappa^{b \rightarrow r} U^{P l}\left(E, T_{e}\right)-\kappa^{r \rightarrow b} U\right] d E .
$$

Скорость обмена энергией между свободными электронами и полем излучения:

$$
Q^{e \rightarrow r}=c \int_{0}^{\infty}\left[\kappa^{e \rightarrow r} U^{P l}\left(E, T_{e}\right)-\kappa^{r \rightarrow e} U\right] d E .
$$

Скорость обмена энергией между веществом в ЛТР и полем излучения:

$$
Q_{L T E}^{e+b \rightarrow r}=Q_{L T E}^{b \rightarrow r}+Q_{L T E}^{e \rightarrow r}=c \int_{0}^{\infty} \kappa_{L T E}^{a b}\left[U^{P l}\left(E, T_{e}\right)-U\right] d E .
$$

Уравнения динамики газа/плазмы (18)-(25) дополняются начальными и граничными условиями.

Излучение в движущейся высокотемпературной плазме. Уравнения баланса (19), (21), (25) учитывают влияние волновой природы света (эффектов Доплера и аберрации света) на обмен энергией-импульсом между излучением и веществом при нерелятивистских скоростях движения $\mathbf{u} / c \ll 1$. Кинетическое уравнение переноса излучения (1) не согласовано с этими уравнениями, т.к. сформулировано для неподвижного вещества. Приведем согласованное слаборелятивистское уравнение переноса излучения в неинерциальной системе отсчета, движущейся вместе с жидкой частицей:

$$
\begin{aligned}
\frac{\rho}{c \frac{d}{d t}\left[\frac{I}{\rho}\right]}+\Omega_{i} \frac{\partial I}{\partial r_{i}}+\frac{\Omega_{i} \Omega_{j}}{c} \frac{\partial u_{j}}{\partial r_{i}}\left[I-\frac{\partial[E I]}{\partial E}\right]-\frac{1}{c} \frac{\partial u_{k}}{\partial r_{i}} \frac{\partial\left[\Omega_{i}\left[\delta_{j k}-\Omega_{j} \Omega_{k}\right] I\right]}{\partial \Omega_{j}}+ \\
+\left[\kappa^{a b}+\kappa^{s c}\right] I=\int_{4 \pi} \mathrm{K}^{s c}\left(\boldsymbol{\Omega} \boldsymbol{\Omega}^{\prime}\right) I\left(\mathbf{\Omega}^{\prime}\right) d \Omega^{\prime}+c \kappa^{e m} \frac{U^{P l}}{4 \pi} .
\end{aligned}
$$


Уравнение выводится из релятивистски инвариантного кинетического уравнения дифференцированием преобразований Лоренца. Из (30) можно получить уравнения для угловых моментов интенсивности:

$$
\begin{aligned}
& \rho \frac{d}{d t}\left[\frac{U(E)}{\rho}\right]+\frac{\partial W_{i}}{\partial r_{i}}+\left[P_{i j}^{r}-\frac{\partial\left[E P_{i j}^{r}\right]}{\partial E}\right] \frac{\partial u_{j}}{\partial r_{i}}+c \kappa^{a b} U=c \kappa^{e m} U^{P l}, \\
& \underline{\underline{\rho}} \frac{d}{d t}\left[\frac{W_{i}(E)}{\rho}\right]+c \frac{\partial P_{i j}^{r}}{\partial r_{j}}+\frac{W_{j}}{c} \frac{\partial u_{i}}{\partial r_{j}}-\frac{1}{c} \frac{\partial\left[E M_{i j k}\right]}{\partial E} \frac{\partial u_{j}}{\partial r_{k}}+\left[\kappa^{a b}+\kappa^{s c}-\kappa_{1}^{s c}\right] W_{i}=0, \\
& U(E)=\frac{1}{c} \int_{4 \pi} I d \boldsymbol{\Omega}, \quad \mathbf{W}(E)=\int_{4 \pi} \boldsymbol{\Omega} I d \mathbf{\Omega}, \quad M_{i j k}(E)=\int_{4 \pi} \Omega_{i} \Omega_{j} \Omega_{k} I d \mathbf{\Omega},
\end{aligned}
$$

а из (31) - уравнения (19), (21), (25).

Рассмотрение круга вопросов, связанных с корректной формулировкой уравнений радиационной газовой динамики и кинетического уравнения переноса излучения, можно найти в работах [27], [32]-[36]. Трансформационные свойства поля излучения следуют из инвариантности тензора энергии-импульса вещества и излучения относительно преобразований Лоренца. Например, поток энергии излучения при изменении системы отсчета преобразуется как:

$$
W_{i}^{L}=W_{i}+u U+u_{j} P_{i j}^{r},
$$

где $\mathbf{W}^{L}$ - поток в системе наблюдателя, $\mathbf{W}, U$ и $P_{i j}^{r}$ - поток энергии, плотность энергии и тензор давления излучения в сопутствующей системе отсчета, движущейся вместе с жидкой частицей со скоростью $\mathbf{u} / c \ll 1$. Преобразование (32), действуя совместно с галилеевым преобразованием потока энергии массовых компонент плазмы, обеспечивает корректный переход от эйлеровой к лагранжевой формулировке уравнения баланса энергии (21).

Необходимость различать лучистые потоки $\mathbf{W}$ и $\mathbf{W}^{L}$ возникает при высоких температурах плазмы, при которых давление излучения становится больше давления электронного газа: $U / 3 \sim a U^{P l} / 3>P_{e}$, где $a$ - число, характеризующее степень ненасыщенности поля излучения. Подставив в неравенство формулы для давлений (6), (20) и оценку среднего заряда $\langle Z\rangle^{2} I_{H} \sim 5 T$, преобразуем его к виду:

$$
T>T_{0}, \quad T_{0,[э B]} \approx 10^{4} \cdot\left[\frac{\rho_{\left[2 \cdot c M^{-3}\right]}}{a\langle A\rangle}\right]^{2 / 5} .
$$

В плазме с нормальной плотностью $\rho \approx 10^{-4} 2 / \mathrm{cm}^{3}$ и $a\langle A\rangle=50$ граничная температура равна $T_{0}=50$ эB. В земных условиях такая плазма может 
возникать при взаимодействии мощных пучков частиц или лазерного излучения с мишенью и при атомном взрыве. В космической плазме с $a\langle A\rangle=1$ и плотностью ниже $\rho \approx 10^{-10} 2 / \mathrm{cm}^{3}$ температура $T_{0}$ становится меньше 1 эB.

Вклады давления излучения помечены в уравнениях (19), (21), (25) нижним подчеркиванием. Этими слагаемыми можно пренебрегать при $T<T_{0}$.

Трудности при моделировании излучающей столкновительной плазмы:

- Многомерность функиий распределения частиц: функция распределения фотонов зависит от семи фазовых переменных $E, \boldsymbol{\Omega}, \mathbf{r}, t$; функция распределения ионов зависит от пяти переменных $E_{n}^{A}, \mathbf{r}, t$.

- Неоднородность и гетерогенность тела: макросечения взаимодействий излучения с веществом могут меняться в пространстве вслед за изменениями плотности и температуры в пределах зоны, состоящей из однородного по элементному составу материала, и испытывать скачки на границах между зонами.

- Изменяемая геометрия тела: размеры зон, профили температуры, плотности и других параметров могут меняться вследствие движения и протекающих нестационарных физических процессов.

- Многорезонансные спектры: зависимость распределения фотонов от энергии несет на себе отпечаток сложной немонотонной зависимости макросечений от энергии, координат и времени.

Для упрощения задачи применяются приближенные методы и приемы. Уменьшения размерности по пространственным переменным можно достигнуть, рассмотрев серию более простых одномерных или двумерных задач. После установления соответствия с исходной задачей (если оно возможно) выполняется оценка решения многомерной задачи. Для уменьшения размерности функции распределения фотонов по угловым переменным используются метод дискретных ординат ( $\mathrm{Sn}-\mathrm{метод)} \mathrm{[56],} \mathrm{[57]} \mathrm{и} \mathrm{разложение}$ распределения по угловым моментам около изотропного распределения - метод сферических гармоник (см. [27], [58]). Часто ограничиваются поиском линейных отклонений - диффузионным приближением [26], [27]. Для уменьшения размерности по энергии используются методы осреднения (агрегации) спектра частиц. В случае спектра энергий ионов это объединение состояний (уровней) с близкой энергией в группы состояний (рассмотрено выше). В случае спектра фотонов - многогрупповое приближение (см. [27], [58]), метод функций пропускания фотонов на участках спектра (см. [2], [11]), приближение Бибермана-Холстейна и вероятностей вылета фотона из тела (см. [28]-[30], [35]-[38]), методы, применяющие элементы интегрования по Лебегу [1]-[21]. Вопросы разделения исходной задачи на итеративное решение нескольких подзадач освещены в [37], [38], [59]-[64]. 


\section{2. Разложение по спектральным моментам}

Эвристическое обоснование разложения. Быстрота сходимости разложения функции по моментам зависит от того, насколько хорошо система базисных функций передает поведение функции. Попробуем угадать «хорошую» систему базисных функций для спектра фотонов из аналитического решения частной задачи. Пусть задача стационарна, рассеяние и отражение фотонов от границы отсутствуют, тепловые источники излучения находятся в равновесии и даются формулой (11), макросечение поглощения не зависит от координат: $\kappa(E)=\eta(E)+\kappa_{\min }$, где $\kappa_{\min }=\min \kappa(E)-$ «фоновое» макросечение. Интегрируя уравнение (1), (3) вдоль характеристики, проходящей через точку наблюдения $\mathbf{r}$ и точку $\mathbf{r}_{\text {in }}$ на границе тела, получим решение:

$$
I(E, l)=I^{e x t}(E) \mathrm{e}^{-\kappa(E)\left[l-l_{i n}\right]}+\frac{c}{4 \pi} \int_{l_{\text {in }}}^{l} \kappa(E) U^{P l}\left(E, l^{\prime}\right) \mathrm{e}^{-\kappa(E)\left[l-l^{\prime}\right]} d l^{\prime} .
$$

Здесь $I$ и $I^{\text {ext }}$ - интенсивность излучения в точках с характеристическими координатами $l$ и $l_{\text {in }}$. Обозначим пройденный путь за $L=l-l_{\text {in }}$. Для пучков, которые пополняются фотонами быстро ( $(E) L \gg 1)$, распределение $U^{P l}\left(E, l^{\prime}\right)$ выносим из-под знака интеграла в точке максимума экспоненты $l^{\prime}=l$ и интегруем остаток. Для пучков, которые пополняются медленно $(\kappa(E) L \ll 1)$, распределение $U^{P l}\left(E, l^{\prime}\right)$ заменяем на среднее $\left\langle U^{P l}\right\rangle$ и тоже выносим из-под знака интеграла. Выполнив сшивку асимптотик, преобразуем (34) к виду:

$$
\begin{gathered}
I(E, l) \approx I^{e x t} \mathrm{e}^{-\kappa L}+\frac{c}{4 \pi}\left[U^{P l}\left[1-\mathrm{e}^{-\kappa L}\right]+\left\langle U^{P l}\right\rangle \mathrm{e}^{-\kappa L}\right]\left[1-\mathrm{e}^{-\kappa L}\right]= \\
=\frac{c U^{P l}(E, l)}{4 \pi} \cdot\left[[1-A-B]+B \cdot \xi+A \cdot \xi^{2}\right], \\
\xi(E)=1-2 e^{-\eta(E) L}, \quad\left\langle U^{P l}\right\rangle=\frac{1}{L} \int_{l_{i n}}^{l} U^{P l} d l^{\prime}, \quad A(E, l)=\frac{e^{-2 \kappa_{\min } L}}{4}\left[1-\frac{\left\langle U^{P l}\right\rangle}{U^{P l}}\right], \\
B(E, l)=\frac{e^{-\kappa_{\min } L}}{2}\left[1-\frac{4 \pi I^{e x t}}{c U^{P l}}+\left[1-e^{-\kappa_{\min } L}\right]\left[1-\frac{\left\langle U^{P l}\right\rangle}{U^{P l}}\right]\right] .
\end{gathered}
$$

Распределение фотонов в гомогенном теле близко к произведению интенсивности источников $c U^{P l} / 4 \pi$ и квадратного трехчлена переменной $\xi$. Переменная $\xi$ может быстро осциллировать по энергии $E$ в пределах интервала $-1 \leq \xi \leq 1$ из-за немонотонного поведения макросечения $\eta(E)=\kappa(E)-\kappa_{\min }$. Коэффициенты $A$ и $B$ меняются с энергией $E$ медленно, если распределение падающих извне фотонов $I^{e x t}$ пропорционально 
интенсивности источников $I^{e x t}(E) / U^{P l}(E, l) \sim \operatorname{const}(l)$ или оба распределения меняются с энергией медленно. Оценка (35) показывает, что в качестве базисных функций разложения можно пробовать степени $\xi^{n}, n=0,1,2, \ldots$

Носители резонансов на шкале энергий фотонов. Для того чтобы сформулировать разложение по спектральным моментам для неоднородного и/или гетерогенного тела, выполним предварительные построения.

Свяжем с каждым состоянием $A, k$ носитель резонансов его макросечения поглощения $\kappa_{A, k}$. Вычислим средние макросечения экстинкции фотонов по объему неоднородного и/или гетерогенного тела $V(t)$ и по характерному временному интервалу $t_{0}<t<t_{0}+\tau$ (интервалам), если задача нестационарна:

$$
\langle\kappa\rangle=\left\langle\kappa^{a b}+\kappa^{s c}\right\rangle=\frac{1}{\langle V\rangle \tau} \int_{t_{0}}^{t_{0}+\tau} \int_{V}\left[\sum_{A, k} \kappa_{A, k}+\kappa^{s c}\right] d \mathbf{r} d t=\sum_{A, k}\left\langle\kappa_{A, k}\right\rangle+\left\langle\kappa^{s c}\right\rangle .
$$

Средние макросечения $\langle\kappa(E)\rangle,\left\langle\kappa_{A, k}(E)\right\rangle$ зависят только от энергии фотонов. Средние макросечения достаточно вычислить приближенно. Погрешность, допускаемая при их расчете, не входит напрямую в погрешность метода спектральных моментов.

Для учета пространственных корреляций между резонансами переходов из состояния иона $A, k$ разобьем спектр энергий на носители резонансов [15], [16]. В носитель резонансов состояния $A, k$ включаются интервалы спектра энергий $E$, на которых его среднее макросечение поглощения больше среднего макросечения любого другого состояния: $\left.\left\langle\kappa_{A, k}(E)\right\rangle\right\rangle\left\langle\kappa_{B, l}(E)\right\rangle$, где $B, l \neq A, k$. Оставшиеся нераспределенными интервалы спектра распределяются по носителям, так что итоговое разбиение плотно покрывает значимый диапазон энергий фотонов. Носитель резонансов может состоять из конечного числа несмежных интервалов. Так как резонансы (линии поглощения) относительно узки по сравнению с расстоянием между ними, то на носителе сильные резонансы поглощения иона в состоянии $A, k$ приходятся преимущественно на фоновое сечение (крылья линий) всех остальных состояний ионов.

Имеет место утверждение. На любом носителе резонансов локальное (в точке $(\mathbf{r}, t))$ макросечение экстинкции близко к линейной функции от среднего макросечения. Например, для носителя резонансов состояния $A, k$ :

$$
\begin{aligned}
& \kappa(E, \mathbf{r}, t)=x\langle\kappa(E)\rangle+y, \\
& x=\frac{\kappa_{A, k}}{\left\langle\kappa_{A, k}\right\rangle}, \quad y=\sum_{B, l \neq A, k}\left[\kappa_{B, l}-x\left\langle\kappa_{B, l}\right\rangle\right]+\kappa^{s c}-x\left\langle\kappa^{s c}\right\rangle .
\end{aligned}
$$

Соотношение нетрудно получить из (36). Здесь $x(E, \mathbf{r}, t)$ и $y(E, \mathbf{r}, t)$ суть функции малой вариации по $E$. Функция $x$ есть дробно-линейная комбинация 
сечений, содержащих одни и те же резонансы (см. (17)). Функция у не содержит резонансов на носителе состояния $A, k$. Если в некоторых зонах гетерогенного тела функция $x$ близка к нулю, то локальное макросечение определяется функцией $y$.

Из (37) следует, что в пределах носителей резонансов распределение фотонов есть функция от среднего макросечения экстинкции $\langle\kappa(E)\rangle$, близкая к функциональной зависимости (35).

Для более точного учета вкладов отдельных переходов $A, k \rightarrow A, l$, $l=l_{1}, l_{2}, \ldots$ (если он требуется) носитель резонансов состояния $A, k$ можно разбить на «вторичные» носители, согласно условию $\left\langle\kappa_{A, k \rightarrow l}(E)\right\rangle>\left\langle\kappa_{A, k \rightarrow j}(E)\right\rangle$, где $j \neq l$.

Число «первичных» носителей резонансов не превышает числа групп состояний ионов $G_{\min } \leq K=M[\Delta Z+1] H$. Число «вторичных» носителей (если они требуются) не превышает числа переходов между группами состояний $G_{\max } \leq[M+1]^{2}[\Delta Z+1] H / 2$. После разбиения шкалы энергий фотонов на носители макросечение поглощения в пределах каждого носителя по-прежнему содержит множество резонансных немонотонностей (линий поглощения). Носители резонансов будем обозначать символом $\omega_{g},(g=1,2, \ldots, G)$. Перенос излучения на каждом носителе рассматривается отдельно.

Весовой спектр. Во многих задачах можно указать грубый, характерный спектр $P(E, \mathbf{r}, t)$, передающий гладкую огибающую спектра фотонов. В задачах переноса теплового излучения в качестве характерного спектра можно взять локальное планковское распределение $U^{P l}\left(E, T_{e}\right)$ (см. (35)), а при наличии сильных изменений температуры - среднее по объему планковское распределение $\left\langle U^{P l}(E)\right\rangle$. В задачах переноса излучения от внешних источников - спектр внешних источников $U^{\text {ext }}(E)$. Если характерный спектр указать затруднительно, то он полагается равным единице. Введем на носителе резонансов $E \in \omega_{g}$ нормированный весовой спектр $S(E, \mathbf{r}, t)$, пропорциональный характерному спектру:

$$
S=P / \int_{\omega_{g}} P d E, \quad \int_{\omega_{g}} S d E=1, \quad\left(P=U^{P l},\left\langle U^{P l}\right\rangle, U^{\text {ext }}\right) .
$$

Разложение распределения по спектральным моментам. Будем искать распределение фотонов в пределах носителя $\omega_{g}$ в виде ряда (сравни с (35)):

$$
I(E, \mathbf{\Omega}, \mathbf{r}, t)=S(E, \mathbf{r}, t) \cdot \sum_{n=0}^{\infty} \frac{P_{n}(\xi)}{d_{n}^{2}} J_{g}^{(n)}(\mathbf{\Omega}, \mathbf{r}, t), \quad\left(E \in \omega_{g}\right),
$$




$$
\begin{aligned}
& \xi(E, \mathbf{\Omega}, \mathbf{r}, t)=1-2 e^{-\eta(E) L} \approx \frac{\eta L-1}{\eta L+1}, \\
& \eta(E)=\langle\kappa(E)\rangle-\kappa_{\min }, \quad \kappa_{\min }=\min _{E \in \omega_{g}}\langle\kappa(E)\rangle,
\end{aligned}
$$

где коффициенты разложения $J_{g}^{(n)}$ (далее - спектральные моменты распределения) не зависят от энергии фотонов, $P_{n}(\xi)$ - система многочленов, ортогональных на отрезке $-1 \leq \xi \leq 1$ с весом $h(\xi)$ :

$$
\int_{-1}^{1} h(\xi) P_{m}(\xi) P_{n}(\xi) d \xi=d_{n}^{2} \delta_{m n}, \quad m, n=0,1,2, \ldots,
$$

$d_{n}$ - нормировочный множитель многочленов. Переменная разложения $\xi(\eta L)$ есть возрастающая функция средней оптической толщины тела $\eta L$, где $\eta(E)-$ среднее макросечение экстинкции фотонов, из которого вычтен «фон» $\kappa_{\min }$, $L(\boldsymbol{\Omega}, \mathbf{r}, t)$ - характерный размер тела. Сечение $\eta(E)$, фон $\kappa_{\min }$ и размер $L$ могут подбираться для каждого носителя резонансов отдельно. Индекс носителя «g» опускаем. Для практических применений представляют интерес два варианта, когда размер $L$ близок:

(1) к длине хорды тела, проходящей через точку $(\mathbf{r}, t)$ в направлении $\boldsymbol{\Omega}$, или к длине характеристики от точки входа до точки выхода пучка из тела: $L \approx l_{\text {out }}-l_{\text {in }}$ (см. Рис. 2);

(2) к среднему диаметру тела безотносительно направлений полета фотонов и координат точки наблюдения: $L \approx\langle L\rangle$.

Разложение (39) эквивалентно разложению по системе функций $\exp (-n \eta(E) L), n=0,1,2, \ldots$, где $\eta(E) L$ - средняя оптическая толщина тела. Наряду с экспоненциальной зависимостью параметра $\xi(\eta L)$ от оптической толщины можно пользоваться ее Паде-аппроксимацией, указанной в (39) знаком приближенного равенства. В этом варианте разложение эквивалентно разложению по системе функций $[\eta(E) L+1]^{-n}$.

Наряду с разложением (39) будем рассматривать разложение планковского распределения и разложение интенсивности внешнего излучения:

$$
\begin{aligned}
& U^{P l}\left(E, T_{e}\right)=S(E, \mathbf{r}, t) \cdot \sum_{n=0}^{\infty} \frac{P_{n}(\xi)}{d_{n}^{2}} U_{g}^{P l(n)}(\mathbf{r}, t), \quad\left(E \in \omega_{g}\right), \\
& I^{\text {ext }}\left(E, \boldsymbol{\Omega}, \mathbf{r}_{i n}, t\right)=S(E, \mathbf{r}, t) \cdot \sum_{n=0}^{\infty} \frac{P_{n}(\xi)}{d_{n}^{2}} J_{g}^{\text {ext }(n)}\left(\boldsymbol{\Omega}, \mathbf{r}_{i n}, t\right) .
\end{aligned}
$$

Если весовой спектр (38) пропорционален планковскому распределению $\left(S(E) \sim U^{P l}\left(E, T_{e}\right)\right)$, то: 


$$
U_{g}^{P l(0)}=\frac{d_{0}^{2}}{P_{0}} \int_{\omega_{g}} U^{P l} d E, \quad U_{g}^{P l(n)}=0, n \geq 1 .
$$

Замена переменной спектра. Аргумент многочленов $\xi(E)$ в разложении (39) есть немонотонная функция энергии $E$. Поэтому для интегрирования ряда по $\xi$, в частности для вычисления спектральных моментов, следует перейти от интегрирования функций по Риману к интегрированию по Лебегу-Стилтьесу. При этом выполняется замена переменной спектра $E$ на новую переменную $m$ - меру лебеговых множеств, относительно которой аргумент разложения $\xi$ становится монотонной функцией. Этими словами можно было бы и ограничиться, отослав читателя к окончательным формулам (51), (52), а за разъяснениями - к литературе по теории функций. Но, так как «прикладные» (выходящие за пределы здания математики) выгоды от интегрирования по Лебегу скудно освещены в литературе, остановимся на них подробнее.

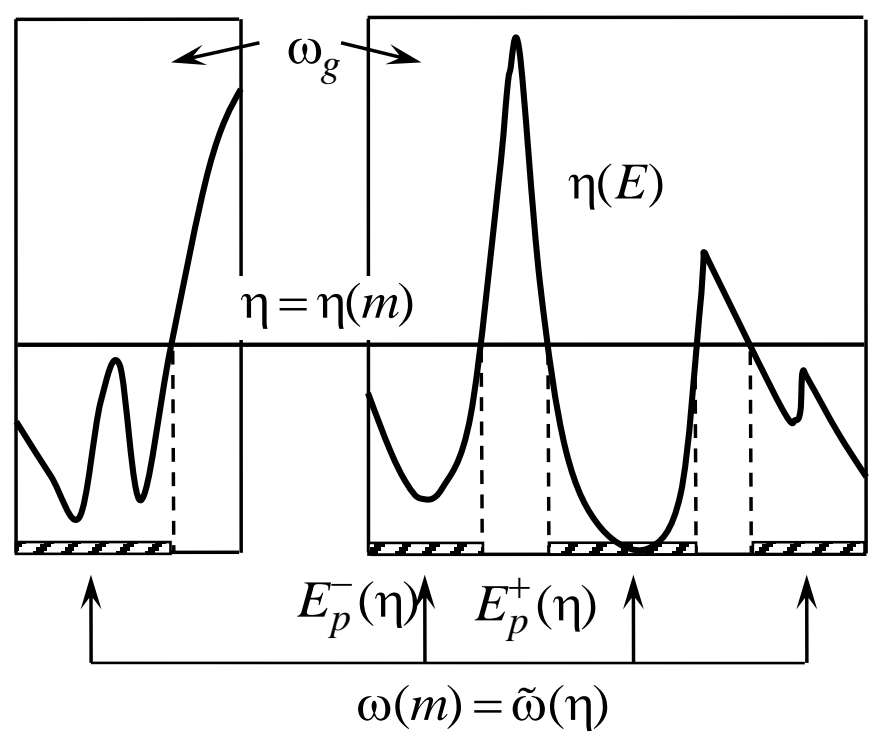

Рис. 3. Носитель резонансов $\omega_{g}$ состоит из двух интервалов спектра, лебегово множество $\omega(m)$ - из четырех интервалов, $\eta(m)$ - уровень поглощения.

Рассмотрим носитель резонансов $E \in \omega_{g}$. Построим систему вложенных друг в друга множеств, включив в множество $\tilde{\omega}(\eta)$ такие энергии $E$, при которых макросечение меньше заданного значения: $\eta(E)<\eta$, где $\eta-$ линия уровня (см. Рис. 3). Множество $\widetilde{\omega}(\eta)$ состоит из серии интервалов $E_{p}^{-}(\eta)<E<E_{p}^{+}(\eta), p=1,2, \ldots$. Левые и правые границы интервалов $E_{p}^{ \pm}(\eta)$ суть точки с равновеликим поглощением: $\eta\left(E_{p}^{ \pm}\right)=\eta$. Очевидно, что $\tilde{\omega}\left(\eta_{1}\right) \subseteq \tilde{\omega}\left(\eta_{2}\right) \subseteq \omega_{g}$ при $\eta_{1}<\eta_{2}$. Полагаем, что функции интегрируемы по всем своим аргументам в интересующей нас области фазового пространства $E \times \boldsymbol{\Omega} \times \mathbf{r} \times t$ по Риману и по Лебегу и эти интегралы равны. Введем меру $m$, $(0 \leq m \leq 1)$ множества $\widetilde{\omega}(\eta)$ с помощью интеграла: 


$$
m(\eta, \mathbf{r}, t)=\int_{\widetilde{\omega}(\eta)} S(E, \mathbf{r}, t) d E=\sum_{p} \int_{E_{p}^{-}(\eta)}^{E_{p}^{+}(\eta)} S(E, \mathbf{r}, t) d E .
$$

Мера есть неубывающая функция параметра $\eta$. Поэтому существует неубывающая обратная функция $\eta=\eta(m, \mathbf{r}, t)$. Выполнив с помощью обратной функции замену переменных $\omega(m, \mathbf{r}, t)=\widetilde{\omega}(\eta(m, \mathbf{r}, t))$, параметризуем систему вложенных множеств через их меру $m$. При $m=0$ лебегово множество пусто $\omega(0)=\varnothing$. При $m=1$ множество $\omega(m)$ всюду плотно заполняет носитель резонансов $g$. Граничные точки множества $E_{p}^{ \pm}(m)=E_{p}^{ \pm}(\eta(m))-$ это точки с равновеликим поглощением уровня $\eta=\eta(m)$.

Дифференцируя (42) по мере множества $m$, получим тождество:

$$
1=\frac{\partial}{\partial m} \sum_{p} \int_{E_{p}^{-}(m)}^{E_{p}^{+}(m)} S(E, \mathbf{r}, t) d E=\sum_{p}\left[S\left(E_{p}, \mathbf{r}, t\right) \frac{\partial E_{p}}{\partial m}\right]_{-}^{+},
$$

где: $\left[S\left(E_{p}\right) \frac{\partial E_{p}}{\partial m}\right]_{-}^{+}=S\left(E_{p}^{+}\right) \frac{\partial E_{p}^{+}}{\partial m}-S\left(E_{p}^{-}\right) \frac{\partial E_{p}^{-}}{\partial m}, \quad \frac{\partial E_{p}^{+}}{\partial m} \geq 0, \frac{\partial E_{p}^{-}}{\partial m} \leq 0$.

Лебегово распределение фотонов. Введем кумулятивное распределение (распределение нарастающим итогом) $F(m, \boldsymbol{\Omega}, \mathbf{r}, t)$ как интеграл от распределения фотонов $I(E, \boldsymbol{\Omega}, \mathbf{r}, t)$ по лебегову множеству $\omega(m)$ :

$$
F(m)=\int_{\omega(m)} I(E) d E=\sum_{p} \int_{E_{p}^{-}(m)}^{E_{p}^{+}(m)} I(E) d E, \quad(0 \leq m \leq 1) .
$$

При $m=0$ кумулятивное распределение равно нулю, при $m=1$ оно равно интегралу от распределения $I(E)$ по носителю резонансов $g$. Частную прозводную $J(m, \boldsymbol{\Omega}, \mathbf{r}, t)=\partial F / \partial m$ будем называть плотностью распределения фотонов на лебеговых множествах $\omega(m)$, или кратко - лебеговым распределением фотонов:

$$
\begin{aligned}
J(m) & =\frac{\partial F}{\partial m}=\frac{\partial}{\partial m} \sum_{p} \int_{E_{p}^{-}(m)}^{E_{p}^{+}(m)} I(E) d E=\sum_{p}\left[I\left(E_{p}\right) \frac{\partial E_{p}}{\partial m}\right]_{-}^{+}= \\
& =\left\langle\frac{I\left(E_{p}^{ \pm}\right)}{S\left(E_{p}^{ \pm}\right)}\right\rangle \cdot \sum_{p}\left[S\left(E_{p}\right) \frac{\partial E_{p}}{\partial m}\right]_{-}^{+}=\left\langle\frac{I\left(E_{p}^{ \pm}\right)}{S\left(E_{p}^{ \pm}\right)}\right\rangle .
\end{aligned}
$$

Последнее равенство получено с помощью (43). Из него следует, что лебегово распределение имеет смысл среднего отношения спектрального распределения 
$I(E)$ к весовому спектру $S(E)$ в граничных точках лебегова множества (точках с равновеликим поглощением) $E_{p}^{ \pm}=E_{p}^{ \pm}(m), p=1,2, \ldots$ Определение среднего корректно, т.к. все слагаемые в суммах неотрицательны. Аргумент $m$ для лебегова распределения $J(m)$ играет роль переменной спектра $E$ для распределения $I(E)$. Наряду с $(45)$, введем плотность планковского распределения на системе лебеговых множеств:

$$
U^{P l}\left(m, T_{e}\right)=\frac{\partial}{\partial m} \sum_{p} \int_{E_{p}^{-}(m)}^{E_{p}^{+}(m)} U^{P l}\left(E, T_{e}\right) d E=\sum_{p}\left[U^{P l}\left(E_{p}\right) \frac{\partial E_{p}}{\partial m}\right]_{-}^{+} .
$$

Если весовой спектр (38) пропорционален планковскому распределению $\left(S(E) \sim U^{P l}\left(E, T_{e}\right)\right)$, то плотность $U^{P l}\left(m, T_{e}\right)$ есть величина постоянная, равная интегралу от планковского распределения по носителю резонансов:

$$
U^{P l}\left(m, T_{e}\right)=U_{g}^{P l}\left(T_{e}\right)=\int_{\omega_{g}} U^{P l}\left(E, T_{e}\right) d E, \quad(0 \leq m \leq 1) .
$$

Вычисление скоростей процессов. Важным прикладным свойством интеграла Лебега является формула вычисления линейных функционалов. Пусть $X(\eta)$ есть произвольная интегрируемая функция, где $\eta=\eta(E)$. Тогда:

$$
\int_{\omega(m)} X(\eta(E)) I(E) d E=\int_{0}^{m} X(\eta(m)) J(m) d m, \quad(0 \leq m \leq 1) .
$$

Равенство доказывается дифференцированием интеграла по $m$, вынесением функции $X\left(\eta\left(E_{p}^{ \pm}\right)\right)$из под знака суммы по $p$ в точках с равновеликим поглощением и обратным интегрированием. Свойство замечательно подходит для вычисления скоростей взаимодействия частиц с веществом на широких интервалах спектра при наличии множественных резонансов [15], [16]. Пусть требуется вычислить интеграл от локального сечения (или макросечения) $\sigma^{v}(E, \mathbf{r}, t)$ некоторой группы процессов $v$ по лебегову множеству $\omega(m) \subseteq \omega_{g}$. Справедливы формулы приближенного лебегова интегрирования:

$$
\begin{gathered}
\frac{\partial}{\partial m} \int_{\omega(m)} \sigma^{v}(E) I(E) d E=\frac{\partial}{\partial m} \sum_{p} \int_{E_{p}^{-}(m)}^{E_{p}^{+}(m)} \sigma^{v}(E) I(E) d E= \\
\quad=\sum_{p}\left[\sigma^{v}\left(E_{p}\right) I\left(E_{p}\right) \frac{\partial E_{p}}{\partial m}\right]_{-}^{+}=\sigma^{v}(I, m) J(m) \approx \sigma^{v}(S, m) J(m), \\
\sigma^{v}(I, m)=\sum_{p}\left[\sigma^{v}\left(E_{p}\right) I\left(E_{p}\right) \frac{\partial E_{p}}{\partial m}\right]_{-}^{+} / \sum_{p}\left[I\left(E_{p}\right) \frac{\partial E_{p}}{\partial m}\right]_{-}^{+},
\end{gathered}
$$




$$
\begin{gathered}
\sigma^{v}(S, m)=\sum_{p}\left[\sigma^{v}\left(E_{p}\right) S\left(E_{p}\right) \frac{\partial E_{p}}{\partial m}\right]_{-}^{+}=\frac{\partial}{\partial m} \int_{\omega(m)} \sigma^{v}(E) S(E) d E, \\
\int_{\omega(m)} \sigma^{v}(E) I(E) d E \approx \int_{0}^{m} \sigma^{v}(S, m) J(m) d m, \quad(0 \leq m \leq 1) .
\end{gathered}
$$

Здесь $\sigma^{v}(I, m, \mathbf{r}, t)$ и $\sigma^{v}(S, m, \mathbf{r}, t)$ суть средние сечения (далее - лебеговы сечения), вычисленные по граничным точкам множества $E_{p}^{ \pm}, p=1,2, \ldots$ (точкам с равновеликим поглощением) соответственно с «неизвестным» распределением фотонов $I(E)$ и с «известным» весовым спектром $S(E)$. Также введем лебегово сечение, вычисляемое с планковским распределением:

$$
\begin{gathered}
\sigma^{v}\left(U^{P l}, m\right)=\sum_{p}\left[\sigma^{v}\left(E_{p}\right) U^{P l}\left(E_{p}\right) \frac{\partial E_{p}}{\partial m}\right]_{-}^{+} / \sum_{p}\left[U^{P l}\left(E_{p}\right) \frac{\partial E_{p}}{\partial m}\right]_{-}^{+}= \\
=\frac{\partial}{\partial m} \int_{\omega(m)} \sigma^{v}(E) U^{P l}(E) d E / \frac{\partial}{\partial m} \int_{\omega(m)} U^{P l}(E) d E, \\
\int_{\omega(m)} \sigma^{v}(E) U^{P l}(E) d E=\int_{0}^{m} \sigma^{v}\left(U^{P l}, m\right) U^{P l}(m) d m, \quad(0 \leq m \leq 1) .
\end{gathered}
$$

Лебеговы сечения $\sigma^{v}(S, m)$ и $\sigma^{v}\left(U^{P l}, m\right)$ можно вычислить заранее, до решения кинетической задачи переноса фотонов. Если весовой спектр пропорционален планковскому распределению, то эти сечения совпадают.

Приближенное лебегово сечение $\sigma^{v}(S, m)$ близко к точному лебегову сечению $\sigma^{v}(I, m)$ в трех случаях:

1) Если весовой спектр пропорционален распределению фотонов $I\left(E_{p}^{ \pm}\right) \approx X \cdot S\left(E_{p}^{ \pm}\right)$в граничных точках множеств в некотором диапазоне меры $\Delta m$. Коэффициентом пропорциональности $X(\eta(E))$ может быть произвольная функция среднего макросечения (она выносится из сумм по $p$ в числителе, знаменателе и сокращается).

2) Если локальное сечение процессов $v$ близко к некоторой функции от среднего макросечения $\sigma^{v}(E) \approx Y(\eta(E))$ или близко к постоянной $\sigma^{v}(E) \approx$ const (тогда сечение $\sigma^{v}\left(E_{p}^{ \pm}\right)$выносится из сумм по $p$ ).

3) Если среднее макросечение $\eta(E)$ есть монотонная функция энергии фотонов в пределах носителя $g$ (тогда в суммах по $p$ отлично от нуля только одно слагаемое). 
В наших предварительных построениях мы старались выполнить условия всех трех случаев. Для выполнения 1) и 2) мы разбили спектр на носители резонансов, на которых локальное макросечение суммы процессов $\kappa(E, \mathbf{r}, t)$ близко к линейной функции (37) от среднего макросечения $\eta(E)$. При этом распределение фотонов оказывается близким к зависимости (35) (случай 1), а локальное сечение группы процессов $\sigma^{v}(E)$ оказывается близким к функции $Y(\eta(E))$, растущей медленнее линейной (случай 2). Случай 3) достигается увеличением количества носителей резонансов.

Связь спектральных моментов с лебеговым распределением фотонов. Если подставить разложение распределения фотонов по спектральным моментам (39) в (45) и воспользоваться (48), мы получим разложение лебегова распределения фотонов:

$$
\begin{aligned}
& J(m, \boldsymbol{\Omega}, \mathbf{r}, t)=\sum_{n=0}^{\infty} \frac{P_{n}(\xi)}{d_{n}^{2}} J_{g}^{(n)}(\boldsymbol{\Omega}, \mathbf{r}, t), \quad\left(\omega(m) \in \omega_{g}\right), \\
& \xi(m)=1-2 e^{-\eta(m) L} \approx \frac{\eta(m) L-1}{\eta(m) L+1}, \quad \eta(m)=\eta\left(E_{p}^{ \pm}(m)\right), \quad(p=1,2, \ldots) .
\end{aligned}
$$

В отличие от разложения (39), в разложении (51) параметр $\xi$ есть неубывающая функция линии уровня $\eta(m)$ и «новой» переменной спектра $m$. Разложение (51) можно интегрировать по переменным $m, \eta(m)$ и $\xi(m)$. Теперь можно дать формальное определение спектральных моментов, входящих в (39) и (51):

$$
J_{g}^{(n)}(\mathbf{\Omega}, \mathbf{r}, t)=\int_{-1}^{1} h(\xi) P_{n}(\xi) J(m(\xi)) d \xi .
$$

Как видим, спектральные моменты суть моменты лебегова распределения фотонов. Если спектральные моменты $J_{g}^{(n)}, 0 \leq n \leq N$, известны (найдены), то функция распределения фотонов внутри тела приближенно восстанавливается по формуле (39) обрыванием ряда на $N$-ом моменте: $J_{g}^{(n)}=0, n \geq N+1$.

Суммарное распределение фотонов на носителе $E \in \omega_{g}$ и скорости процессов, описываемых сечением $\sigma^{v}(E, \mathbf{r}, t)$, вычисляются по формулам:

$$
\begin{aligned}
& I_{g}(\mathbf{\Omega}, \mathbf{r}, t)=\int_{\omega_{g}} I(E, \mathbf{\Omega}, \mathbf{r}, t) d E \approx \sum_{n=0}^{N} T_{g}^{(n)} J_{g}^{(n)}, \\
& \int_{\omega_{g}} \sigma^{v}(E) I(E) d E \approx \sum_{n=0}^{N} \sigma_{g}^{v,(n)} T_{g}^{(n)} J_{g}^{(n)}
\end{aligned}
$$




$$
\begin{aligned}
& \int_{\omega_{g}} \sigma^{v}(E) U^{P l}(E) d E \approx \sum_{n=0}^{N} \sigma_{g}^{v,(n)} T_{g}^{(n)} U_{g}^{P l(n)}, \\
& T_{g}^{(n)}=\int_{\omega_{g}} \frac{P_{n}(\xi(E))}{d_{n}^{2}} S(E) d E, \quad \sigma_{g}^{v,(n)} T_{g}^{(n)}=\int_{\omega_{g}} \sigma^{v}(E) \frac{P_{n}(\xi(E))}{d_{n}^{2}} S(E) d E .
\end{aligned}
$$

Здесь мы воспользовались разложением (39). Таблицы функций $T_{g}^{(n)}(\mathbf{r}, t, L)$ и сечений $\sigma_{g}^{v,(n)}(\mathbf{r}, t, L)$ можно вычислить заранее, до решения задачи.

\section{3. Вычисление спектральных моментов}

Способы вычисления спектральных моментов. Существует по крайней мере два способа вычисления спектральных моментов распределения. Первый, традиционный способ, состоит в формулировке системы уравнений относительно моментов и нахождении ее решения. Такая система моментных уравнений сформулирована автором в [65]. Второй способ, который мы применим ниже, использует квадратурную формулу Гаусса. Он приводит к конечному результату быстрее и при меньших ограничениях (в [65] применялась дробно-линейная аппроксимация параметра $\xi$ (см. (39)).

Квадратурная формула Гаусса-Кристоффеля имеет вид (см., напр., [66]):

$$
\int_{-1}^{1} h(\xi) F(\xi) d \xi \approx \sum_{i=0}^{N} \lambda_{i} F\left(\xi_{i}\right), \quad \sum_{i=0}^{N} \lambda_{i}=\int_{-1}^{1} h(\xi) d \xi .
$$

Узлы квадратуры $\xi_{i}$ определяются нулями многочлена степени $N+1$ $\left(P_{N+1}\left(\xi_{i}\right)=0\right)$, а весовые коэффициенты Кристоффеля $\lambda_{i}$ вычисляются как

$$
\frac{1}{\lambda_{i}}=\sum_{n=0}^{N} \frac{P_{n}^{2}\left(\xi_{i}\right)}{d_{n}^{2}}=\frac{a_{N} P_{N}\left(\xi_{i}\right)}{a_{N+1} d_{N}^{2}} \frac{d P_{N+1}\left(\xi_{i}\right)}{d \xi} .
$$

Здесь $h(\xi)$ - весовая функция, $F(\xi)$ - интегрируемая функция, $F\left(\xi_{i}\right)$ значения интегрируемой функции в узлах $\xi_{i},(i=0,1, \ldots, N), a_{N}$ - коэффициент при старшей степени многочлена $P_{N}$. Формула строится так, что приближенное равенство переходит в точное для любых многочленов степени не выше $2 N+1$.

Рассмотрим приближение $J_{N}(m)$ к лебегову распределению фотонов $J(m)$ в виде отрезка ряда (51) длиной $N+1$ :

$$
J_{N}(m)=\sum_{n=0}^{N} \frac{P_{n}(\xi)}{d_{n}^{2}} J_{g}^{(n)} \approx J(m) .
$$

$N$ называется порядком метода моментов. Точное распределение и его приближение имеют одинаковые моменты $J_{g}^{(n)}, 0 \leq n \leq N$. Так как функция 
$F(\xi)=P_{n}(\xi) J_{N}(m)$ при $0 \leq n \leq N$ есть многочлен степени не выше $2 N$, то ее подстановка в (54) приводит к точной формуле вычисления моментов:

$$
J_{g}^{(n)}(\mathbf{\Omega}, \mathbf{r}, t)=\int_{-1}^{1} h(\xi) P_{n}(\xi) J(m(\xi)) d \xi=\sum_{i=0}^{N} \lambda_{i} P_{n}\left(\xi_{i}\right) J\left(m_{i}\right) .
$$

Все, что осталось сделать, так это найти лебегово распределение фотонов $J\left(m_{i}, \boldsymbol{\Omega}, \mathbf{r}, t\right)$ в $N+1-$ ой точках спектра $m_{i}$, соответствующих узлам формулы Гаусса-Кристоффеля:

$$
m_{i}=m\left(\eta_{i}\right), \quad \eta_{i}=-\frac{1}{L} \ln \frac{1-\xi_{i}}{2} \approx \frac{1}{L} \frac{1+\xi_{i}}{1-\xi_{i}}, \quad(0 \leq i \leq N) .
$$

Знак приближенного равенства относится к варианту, когда выполняется дробно-линейная аппроксимация параметра $\xi$ (см. (39)). Если параметр разложения $L$ выбирается постоянным для тела (см. комментарий к (39)), то постоянны и значения $\eta_{i}, m_{i}$. Если параметр $L$ выбирается индивидуально для характеристик, то значения $\eta_{i}, m_{i}$ могут быть разными на разных характеристиках.

Для примера приведем формулы вычисления спектральных моментов при разложении распределения фотонов по многочленам Чебышева:

$$
P_{n}(\xi)=\cos (n \arccos \xi), \quad h(\xi)=\frac{1}{\sqrt{1-\xi^{2}}}, \quad \int_{-1}^{1} \frac{P_{n} P_{m}}{\sqrt{1-\xi^{2}}} d \xi=d_{n}^{2} \delta_{n m}, \quad d_{n}^{2}=\frac{\pi}{2}\left[1+\delta_{n 0}\right] .
$$

Узлы, значения полиномов в узлах и веса квадратурной формулы (54):

$$
\xi_{i}=\cos \frac{\pi[2 i+1]}{2[N+1]}, \quad P_{n}\left(\xi_{i}\right)=\cos \frac{\pi n[2 i+1]}{2[N+1]}, \quad \lambda_{i}=\frac{\pi}{N+1}, \quad(0 \leq i, n \leq N) .
$$

Формула (55) для вычисления спектральных моментов:

$$
J_{g}^{(n)}(\boldsymbol{\Omega}, \mathbf{r}, t)=\frac{\pi}{N+1} \sum_{i=0}^{N} \cos \frac{\pi n[2 i+1]}{2[N+1]} J\left(m_{i}\right), \quad\left(\omega(m) \in \omega_{g}\right) .
$$

Уравнение (56) для определения дискретных значений меры множеств $m_{i}=m\left(\eta_{i}\right)$ :

$$
\eta_{i}=-\frac{2}{L} \cdot \ln \left(\sin \frac{\pi[2 i+1]}{4[N+1]}\right) \approx \frac{1}{L} \tanh ^{-2} \frac{\pi[2 i+1]}{4[N+1]}, \quad(0 \leq i \leq N) .
$$

Кинетическое уравнение для лебегова распределения фотонов. Расчет лебегова распределения фотонов $J\left(m_{i}, \boldsymbol{\Omega}, \mathbf{r}, t\right)$ в точках $m_{i}$ проще всего 
выполнить с помощью кинетического уравнения, сформулированного в терминах распределения $J(m, \boldsymbol{\Omega}, \mathbf{r}, t)$. Для вывода этого уравнения в нашем распоряжении имеется все необходимое. Проинтегрируем уравнение переноса излучения (30) по лебегову множеству $E \in \omega(m, \mathbf{r}, t) \subseteq \omega_{g}$, учтем определения лебегова распределения фотонов (44)-(46), лебеговых сечений (49), (50) и продифференцируем результат по параметру $m$ :

$$
\begin{array}{r}
\frac{\frac{\rho}{c} \frac{d}{d t}\left[\frac{J}{\rho}\right]}{}+\Omega_{i} \frac{\partial J}{\partial r_{i}}+\frac{\frac{\Omega_{i} \Omega_{j}}{c} \frac{\partial u_{j}}{\partial r_{i}}\left[J-\frac{\partial[m J]}{\partial m}\right]-\frac{1}{c} \frac{\partial u_{k}}{\partial r_{i}} \frac{\partial\left[\Omega_{i}\left[\delta_{j k}-\Omega_{j} \Omega_{k}\right] J\right]}{\partial \Omega_{j}}+}{+\kappa(S, m) J}=\int_{4 \pi} \mathrm{K}^{s c}\left(S, m, \boldsymbol{\Omega} \boldsymbol{\Omega}^{\prime}\right) J\left(\boldsymbol{\Omega}^{\prime}\right) d \Omega^{\prime}+c \kappa^{e m} \frac{U^{P l}(m)}{4 \pi}+\frac{\partial[A J]}{\partial m} .
\end{array}
$$

Аналогично поступим с граничными условиями (3):

$$
\left.J\left(m, \boldsymbol{\Omega}, \mathbf{r}_{i n}\right)\right|_{\mathbf{n} \mathbf{\Omega}<0}=\int_{\mathbf{n} \boldsymbol{\Omega}^{\prime}>0} \Sigma\left(S, m, \boldsymbol{\Omega}^{\prime} \rightarrow \boldsymbol{\Omega}\right) J\left(m, \boldsymbol{\Omega}^{\prime}\right) d \boldsymbol{\Omega}^{\prime}+\left.J^{e x t}\left(m, \boldsymbol{\Omega}, \mathbf{r}_{i n}\right)\right|_{\mathbf{n} \mathbf{\Omega}<0} .
$$

Коэффициенты уравнения равны:

$$
\begin{aligned}
& \kappa(S, m)=\kappa^{a b}(S, m)+\kappa^{s c}(S, m), \quad\left(\omega(m, \mathbf{r}, t) \subseteq \omega_{g}\right) \\
& \kappa^{a b}(S, m)=\sum_{A, k} N_{k}^{A}\left[\sum_{l \geq k}\left[1+\frac{\left.1-N_{l}^{A} \bar{N}_{k}^{A} / N_{k}^{A} \bar{N}_{l}^{A}\right]}{e^{\left[E_{l}^{A}-E_{k}^{A}\right] / T_{e}}-1}\right] \sigma_{k \rightarrow l}^{A}(S, m)+N_{e} \sigma_{k, e \rightarrow e}^{A}(S, m)\right] \\
& \kappa^{e m}\left(U^{P l}, m\right)=\sum_{A, k}\left[\sum_{l \geq k} N_{l}^{A} \frac{\bar{N}_{k}^{A}}{\bar{N}_{l}^{A}} \sigma_{k \rightarrow l}^{A}\left(U^{P l}, m\right)+N_{e} N_{k}^{A} \sigma_{k, e \rightarrow e}^{A}\left(U^{P l}, m\right)\right] \\
& A(S, m, \mathbf{\Omega}, \mathbf{r}, t)=-\int_{\omega(m)} \frac{d S}{d l} d E \approx-\int_{\omega(m)} \mathbf{\Omega} \frac{\partial S}{\partial \mathbf{r}} d E
\end{aligned}
$$

Лебеговы сечения $\sigma_{k \rightarrow l}^{A}(S, m), \quad \sigma_{k \rightarrow l}^{A}\left(U^{P l}, m\right), \quad \sigma_{k, e \rightarrow e}^{A}(S, m), \quad \sigma_{k, e \rightarrow e}^{A}\left(U^{P l}, m\right)$, $\kappa^{s c}(S, m), \mathrm{K}^{s c}(S, m), \Sigma(S, m)$ вычисляются по формулам (49), (50). $A(S, m)$ корректор, учитывающий изменения весового спектра в пространстве. Обычно он пренебрежимо мал вследствие нормировки (38).

Уравнение (57) несколько отличается от уравнения, полученного в работах автора [14]-[16], способом построения лебеговых множеств и наличием подчеркнутых слагаемых, учитывающих движение среды. Напомним, что подчеркнутыми слагаемыми можно пренебрегать при температурах $T<T_{0}$ (см. комментарий к формуле (33)).

Приведем уравнения для угловых моментов распределения фотонов: 


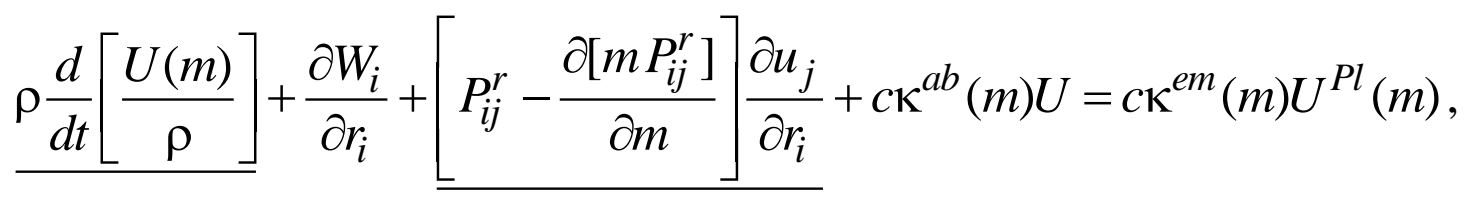

$$
\begin{aligned}
& \underline{\underline{\rho}} \frac{d}{d t}\left[\frac{W_{i}(m)}{\rho}\right]+c \frac{\partial P_{i j}^{r}}{\partial r_{j}}+\frac{W_{j}}{c} \frac{\partial u_{i}}{\partial r_{j}}-\frac{1}{c} \frac{\partial\left[m M_{i j k}\right]}{\partial m} \frac{\partial u_{j}}{\partial r_{k}}+\left[\kappa^{a b}+\kappa^{s c}-\kappa_{1}^{s c}\right] W_{i}=0, \\
& U(m)=\frac{1}{c} \int_{4 \pi} J d \boldsymbol{\Omega}, \quad \mathbf{W}(m)=\int_{4 \pi} \boldsymbol{\Omega} J d \boldsymbol{\Omega}, \quad M_{i j k}(m)=\int_{4 \pi} \Omega_{i} \Omega_{j} \Omega_{k} J d \boldsymbol{\Omega} .
\end{aligned}
$$

При использовании метода квазидиффузии [59]-[62], основанного на независимом решении системы (59), система дополняется условиями:

$$
\begin{aligned}
& \mathbf{n W}\left(m, \mathbf{r}_{i n}\right)=\frac{1-\chi}{1+\chi} \cdot d \cdot c U\left(m, \mathbf{r}_{i n}\right)+\frac{2}{1+\chi} \cdot \mathbf{n} \mathbf{W}^{e x t}\left(m, \mathbf{r}_{i n}\right), \\
& d\left(m, \mathbf{r}_{i n}\right)=\int_{4 \pi}|\mathbf{n} \boldsymbol{\Omega}| I(\boldsymbol{\Omega}) d \mathbf{\Omega} / \int_{4 \pi} I(\boldsymbol{\Omega}) d \mathbf{\Omega}, \quad \mathbf{n} \mathbf{W}^{e x t}\left(m, \mathbf{r}_{i n}\right)=-\int_{\mathbf{n} \mathbf{\Omega}<0}|\mathbf{n} \boldsymbol{\Omega}| I^{e x t}(\boldsymbol{\Omega}) d \mathbf{\Omega} . \\
& \chi\left(m, \mathbf{r}_{i n}\right)=\int_{\mathbf{n} \boldsymbol{\Omega}<0} d \boldsymbol{\Omega} \int_{\mathbf{n} \mathbf{\Omega}^{\prime}>0}|\mathbf{n} \boldsymbol{\Omega}| \Sigma\left(\boldsymbol{\Omega}^{\prime} \rightarrow \boldsymbol{\Omega}\right) I\left(\boldsymbol{\Omega}^{\prime}\right) d \mathbf{\Omega}^{\prime} / \int_{\mathbf{n} \mathbf{\Omega}^{\prime}>0} \mathbf{n} \boldsymbol{\Omega}^{\prime} I\left(\boldsymbol{\Omega}^{\prime}\right) d \mathbf{\Omega}^{\prime} .
\end{aligned}
$$

Уравнения поуровневой кинетики. Концентрации возбужденных состояний находятся из системы уравнений кинетики, записанной через спектральные моменты излучения:

$$
\begin{gathered}
N_{A} \frac{d}{d t}\left[\frac{E_{k}^{A} N_{k}^{A}}{N_{A}}\right]=\sum_{l>k}\left[C_{k \rightarrow l}^{A, e \rightarrow b} \frac{E_{l}^{A}-E_{k}^{A}}{2}-C_{k \rightarrow l}^{A+} \frac{E_{l}^{A}+E_{k}^{A}}{2}\right]\left[N_{k}^{A}-N_{l}^{A} \frac{\bar{N}_{k}^{A}}{\bar{N}_{l}^{A}}\right]+\quad(61) \\
+\sum_{l<k}\left[C_{l \rightarrow k}^{A, e \rightarrow b} \frac{E_{k}^{A}-E_{l}^{A}}{2}+C_{l \rightarrow k}^{A+} \frac{\left.E_{l}^{A}+E_{k}^{A}\right]}{2}\right]\left[N_{l}^{A}-N_{k}^{A} \frac{\bar{N}_{l}^{A}}{\bar{N}_{k}^{A}}\right]+ \\
+\frac{c}{Y} \sum_{g, n} T_{g}^{(n)} \sum_{l \geq k} \frac{\sigma_{k \rightarrow l, g}^{A, r \rightarrow b,(n)}-\sigma_{k \rightarrow l, g}^{A+,(n)}}{2}\left[N_{k}^{A} U_{g}^{(n)}\left[1+\frac{1-N_{l}^{A} \bar{N}_{k}^{A} / N_{k}^{A} \bar{N}_{l}^{A}}{e^{\left[E_{l}^{A}-E_{k}^{A}\right] / T_{e}}-1}\right]-N_{l}^{A} \frac{\bar{N}_{k}^{A}}{\bar{N}_{l}^{A}} U_{g}^{P l(n)}\right]+ \\
+\frac{c}{Y} \sum_{g, n} T_{g}^{(n)} \sum_{l \leq k} \frac{\sigma_{l \rightarrow k, g}^{A, r \rightarrow b,(n)}+\sigma_{l \rightarrow k, g}^{A+,(n)}}{2}\left[N_{l}^{A} U_{g}^{(n)}\left[1+\frac{1-N_{k}^{A} \bar{N}_{l}^{A} / N_{l}^{A} \bar{N}_{k}^{A}}{e^{\left[E_{k}^{A}-E_{l}^{A}\right] / T_{e}}-1}\right]-N_{k}^{A} \frac{\bar{N}_{l}^{A}}{\bar{N}_{k}^{A}} U_{g}^{P l(n)}\right] .
\end{gathered}
$$

Функции $T_{g}^{(n)}$ и сечения $\sigma_{k \rightarrow l, g}^{A, r \rightarrow b,(n)}, \sigma_{k \rightarrow l, g}^{A+,(n)}, \quad(0 \leq n \leq N)$ вычисляются по формулам (53).

Кинетические уравнения (57)-(61) являются основными уравнениями метода. Совместное решение уравнения переноса излучения (57), (58) в узлах 
$m_{i}, \quad 0 \leq i \leq N$ и системы уравнений поуровневой кинетики (61) ищется итерациями по значениям спектральных моментов, вычисляемых по формуле (55). Для интерполяции лебегова распределения $J(m, \mathbf{\Omega}, \mathbf{r}, t)$ по переменной $m$ используется формула (51). Лебеговы сечения вычисляются заранее, до решения кинетической задачи и хранятся в табличных массивах.

\section{4. Сходимость разложения}

Описание тестовой задачи. Продемонстрируем скорость сходимости метода спектральных моментов на задаче формирования стационарного распределения фотонов в плоском слое $0 \leq x \leq 1 \mathrm{~cm}$ в предположении, что вещество находится в ЛТР. Температура падает линейно от значения $T(0)=1.4$ э $B$ на левой границе слоя до значения $T(1)=0.28$ э $B$ на правой границе, при общем падении в 5 раз. Тепловые источники излучения задаются планковским распределением (11). Падение интенсивности источников $c \int U^{P l}\left(E, T_{e}\right) d E=4 \sigma T_{e}^{4}(x)$ составляет $5^{4}=625$ раз. Геометрические параметры тестовой задачи характерны для ударного слоя, возникающего вблизи поверхности космического аппарата на участке торможения в атмосфере Земли. Скорость поглощения и испускания фотонов веществом задается модельным коэффициентом Эльзассера [67]:

$$
\kappa(E, x)=\sum_{j=-\infty}^{\infty} \frac{D \gamma / \pi}{[E-\delta-j \varepsilon]^{2}+\gamma^{2}}=\frac{D \sinh (2 \pi \gamma / \varepsilon) / \varepsilon}{\cosh (2 \pi \gamma / \varepsilon)-\cos (2 \pi[E-\delta] / \varepsilon)} .
$$

Макросечение формируется серией одинаковых линий с постоянным шагом $\varepsilon$, силой $D(x)$ и лоренцевским контуром с полушириной $\gamma(x)$ и сдвигом $\delta(x)$. Расстояние между линиями много меньше температуры вещества $\varepsilon \ll T$. Модельный коэффициент выбран намеренно, с тем чтобы результаты теста не носили характер «уникальных», а могли быть легко воспроизведены в независимом расчете. Лебегово макросечение $\kappa(m, x)$ и планковский коэффициент $\kappa_{P}(x)$ (характеризует среднюю скорость испускания излучения веществом) для сечения (62) на любом широком носителе резонансов $\omega_{g}=\left\{E_{g-1}<E<E_{g}\right\}, E_{g}-E_{g-1} \gg \varepsilon$ даются аналитическими формулами:

$$
\kappa(m, x) \approx \frac{\kappa_{P} \sinh (2 \pi \gamma / \varepsilon)}{\cosh (2 \pi \gamma / \varepsilon)+\cos (\pi m)}, \quad \kappa_{P}(x)=\int \kappa(E) S(E) d E \approx \frac{D}{\varepsilon} .
$$

Коэффициенты не зависят ни от ширины носителя резонансов, ни от положений его границ, поэтому они справедливы и для носителей, состоящих из нескольких интервалов, и для носителя $\omega_{g}=\{0 \leq E<\infty\}$, охватывающего весь спектр.

Неоднородный гомогенный слой. Результаты тестов показали быструю сходимость метода спектральных моментов к точному решению. На Рис. 4 
приведены графики плотности энергии излучения (2) в зависимости от координаты $x$ при трех значениях средней оптической толщины слоя $\kappa_{P} \Delta x=2,10$ и 50. $U(x)$ - точное решение, $U_{N}(x)$ - расчеты в $N-$ приближениях метода моментов. Плотность энергии дана в единицах $\sigma / c=1$. Кривые, совпавшие с кривой точного решения, не представлены. Точная функция распределения фотонов находилась прямым численным решением спектрального уравнения переноса (1)-(3) на подробной сетке по энергии фотонов. В методе спектральных моментов использовались разложение по многочленам Чебышева с параметром $L=1.35 \mathrm{~cm}$ (характерный размер тела) одинаковым для всех направлений полета фотонов, и решения кинетического уравнения (57)-(58). Пространственные профили планковского коэффициента, отношения полуширины линии к расстоянию между линиями $\gamma / \varepsilon=1.6 \cdot 10^{-4}$ и сдвиг линий $\delta=0$ задавались постоянными.
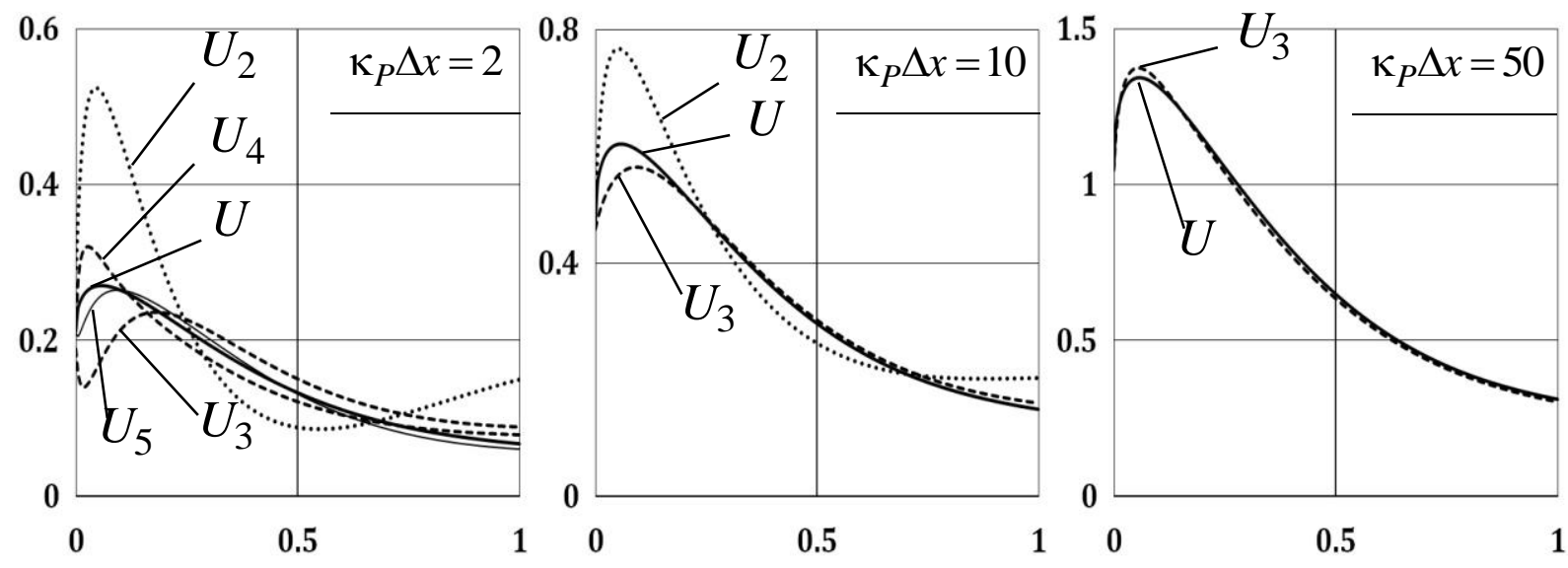

Рис. 4. Сходимость плотности энергии излучения $U_{N}(x)$ $\kappa$ точному решению $U(x)$.

Для оценки скорости сходимости моментного разложения к точному решению подсчитывалась относительная энергия $H_{n},\left(\sum H_{n}=1\right)$, запасенная в моментах $n$, и относительная энергия $G_{n}$ хвоста разложения:

$$
H_{n}(\boldsymbol{\Omega}, x)=\frac{J^{(n) 2} / d_{n}^{2}}{\sum_{k=0} J^{(k) 2} / d_{k}^{2}}, \quad G_{n}(\boldsymbol{\Omega}, x)=\frac{\sum_{k=n} J^{(k) 2} / d_{k}^{2}}{\sum_{k=0} J^{(k) 2} / d_{k}^{2}} .
$$

Распределение энергии $\left\langle H_{n}\right\rangle$ по моментам $n$ для разложения $N=6$, $L=1.35 \mathrm{~cm}$ в слое $\kappa_{P} \Delta x=10$ приведено на Рис. 5. Скобки означают, что распределение осреднено по направлениям полета фотонов и объему тела.

Чувствительность к выбору параметра $L$. Удивительной оказалась слабая чувствительность скорости сходимости моментного разложения к выбору параметра разложения L. Это демонстрирует Рис. 6, на котором 
приведена энергия хвоста разложения $\left\langle G_{3}\right\rangle$ как функция $L$ в слое $\kappa_{P} \Delta x=10$. При уменьшении или увеличении параметра от значения $L=1.35 \mathrm{~cm}$ в 10 раз энергия хвоста увеличивается не более чем в три раза и не превышает $12 \%$.

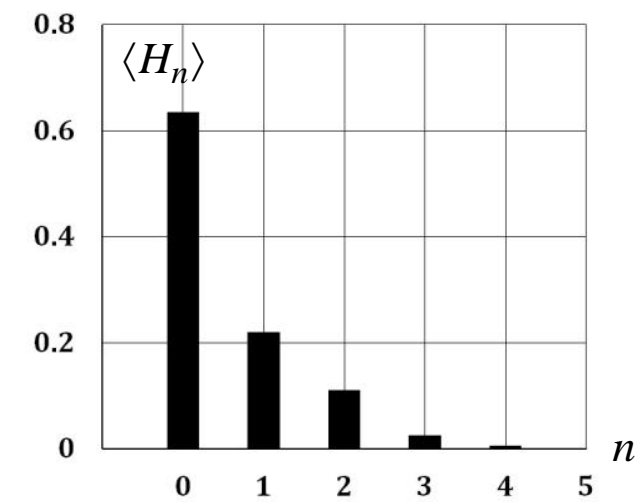

Рис. 5. Распределение энергии $\left\langle H_{n}\right\rangle$ по моментам «n».

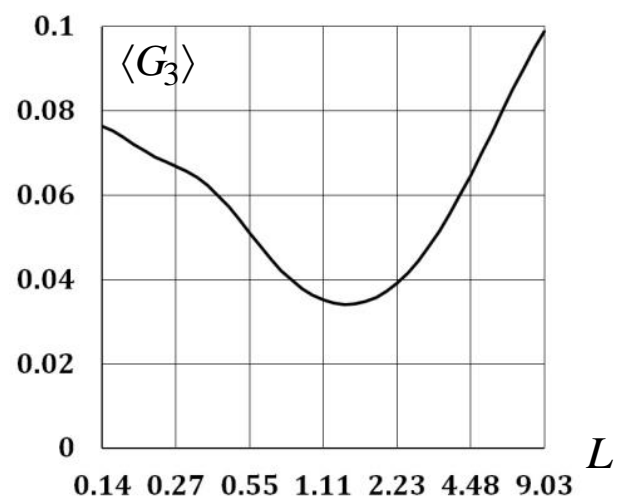

Рис. 6. Энергия хвоста разложения

$\left\langle G_{3}\right\rangle$ как функиия параметра $L$.

Гетерогенный слой. Тестирование метода для гетерогенного тела проводилось на той же серии задач. Единственное отличие состоит в том, что в одной половине слоя $0 \leq x<0.5 \mathrm{~cm}$ вещество имеет макросечение поглощения (62), а во второй половине $0.5 \mathrm{~cm}<x \leq 1 \mathrm{~cm}$ - макросечение, линии которого смещены на половину периода $\delta=\varepsilon / 2$. При этом формальное произведение планковского коэффициента на ширину слоя $\kappa_{P} \Delta x=2,10,50$ не меняется, хотя реальная оптическая толщина гетерогенного слоя примерно в два раза меньше. При выполнении расчетов методом моментов спектр энергий фотонов был поделен на два носителя резонансов. Метод показал такую же скорость сходимости, как и в серии задач для гомогенного слоя (см. Рис. 7).
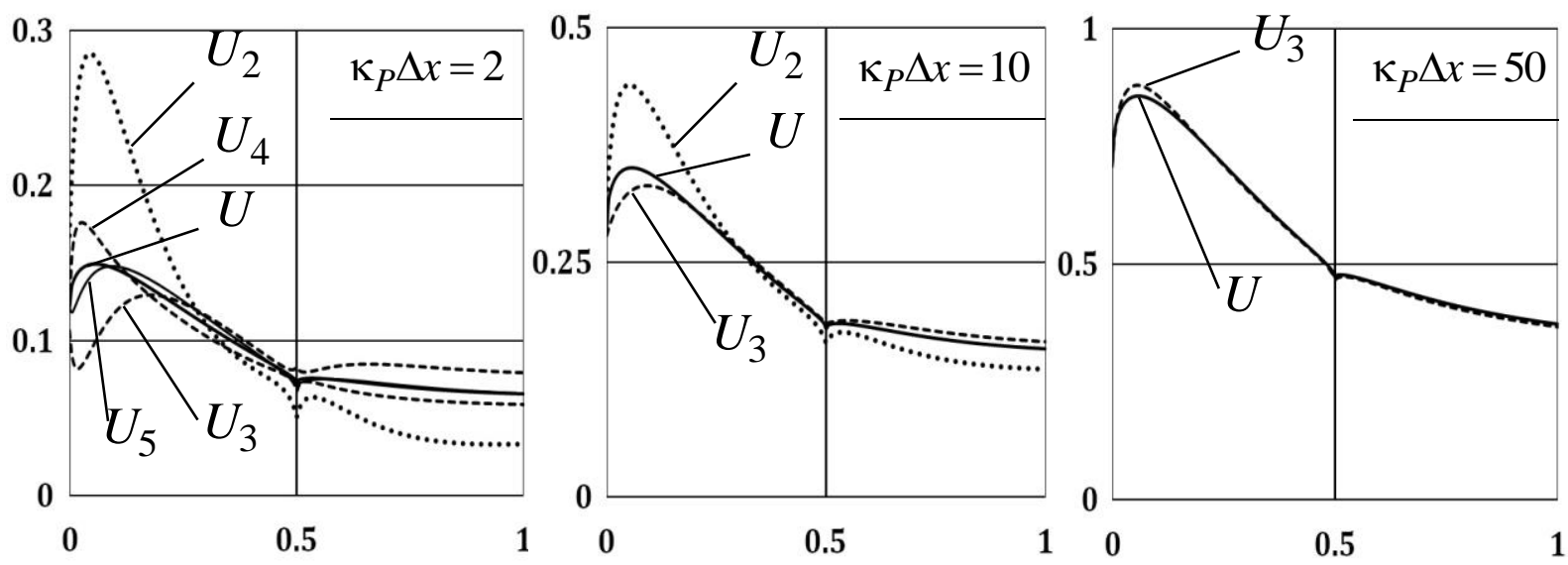

Рис. 7. Сходимость плотности энергии излучения $U_{N}(x)$ $\kappa$ точному решению $U(x)$ в гетерогенном слое.

Резюме результатов. Расчеты задач показали, что метод спектральных моментов при разложении по многочленам Чебышева в варианте (2), когда 
параметр $L$ полагается равным среднему диаметру тела $\langle L\rangle$, воспроизводит решение практически точно в порядке метода $N=4 \div 6$. Расчеты в варианте (1), когда параметр $L$ подбирается индивидуально для каждой характеристики, показали чуть более высокую скорость сходимости - в порядке $N=3 \div 5$.

Расчеты с использованием разложения по многочленам Лежандра показали несколько более медленную сходимость - в порядке $N=6 \div 8$.

\section{5. Примерная методика численного решения}

Основным принципом построения методик численного решения задач радационной газовой динамики является разделение исходной задачи на ряд подзадач с помощью вычисления и «замораживания» кинетических коэффициентов и некоторых физических величин на шаге (шагах) по времени или на шаге итераций [6], [59], [61], [62]-[64]. В случае сильной нелинейности задачи также применяется линеаризация подзадач относительно приращений искомых величин. Тогда на временном шаге или шаге итераций замораживаются не только кинетические коэффициенты, но и их линейные отклики на приращения искомых величин (производные Фреше).

На этом же принципе может быть построена и методика моделирования излучающей столкновительной плазмы с использованием метода спектральных моментов (см. Рис. 8). Компьютерный код решает следующие задачи.

Задача «G» - рассчитывать динамику и геометрию течения. Решаются уравнение неразрывности (18) и уравнения движения (19) при заданном (замороженном) поле давления и внешних источников импульса. Входными данными задачи являются поле давления компонент плазмы. Выходными данными - поля плотности и газодинамической скорости. В лагранжевом представлении движения здесь же рассчитываются координаты ячеек сетки.

Задача «Т» - рассчитывать перераспределение энергии в системе. Решаются: система уравнений баланса кинетической энергии ионов (22) относительно кинетической температуры ионов; система уравнений баланса энергии электронов (23) относительно температуры электронов; уравнение для внутренней энергии ионов (24) относительно внутренней энергии ионов и система уравнений баланса энергии излучения (25) относительно плотности и потока энергии излучения.

Скорости обмена энергией между компонентами плазмы (26)-(29) в уравнениях динамики плазмы (23)-(25) выражаются через отклонения энергий от их равновесных значений и кинетические коэффициенты.

Скорость обмена энергией между свободными электронами и внутренней энергией ионов в безызлучательных столкновениях:

$$
Q^{e \rightarrow b}=C_{t o t}^{e \rightarrow b} \rho \overline{\varepsilon_{b}}-C_{t o t}^{b \rightarrow e} \rho \varepsilon_{b},
$$




$$
\begin{gathered}
C_{t o t}^{e \rightarrow b}=\frac{\sum_{A, k} N_{k}^{A} \sum_{l>k} C_{k \rightarrow l}^{A, e \rightarrow b}\left[E_{l}^{A}-E_{k}^{A}\right]}{\sum_{A, l} \bar{N}_{l}^{A} E_{l}^{A}}=\frac{\sum_{A, l} \bar{N}_{l}^{A} \sum_{k<l} \frac{N_{k}^{A}}{N_{k}^{A}} C_{l \rightarrow k}^{A, e \rightarrow b}\left[E_{l}^{A}-E_{k}^{A}\right]}{\sum_{A, l} \bar{N}_{l}^{A} E_{l}^{A}}, \\
C_{\text {tot }}^{b \rightarrow e}=\frac{\sum_{A, k} \bar{N}_{k}^{A} \sum_{l>k} \frac{N_{l}^{A}}{\bar{N}_{l}^{A}} C_{k \rightarrow l}^{A, e \rightarrow b}\left[E_{l}^{A}-E_{k}^{A}\right]}{\sum_{A, l} N_{l}^{A} E_{l}^{A}}=\frac{\sum_{A, l} N_{l}^{A} \sum_{k<l} C_{l \rightarrow k}^{A, e \rightarrow b}\left[E_{l}^{A}-E_{k}^{A}\right]}{\sum_{A, l} N_{l}^{A} E_{l}^{A}} .
\end{gathered}
$$

Здесь $\rho \overline{\varepsilon_{b}}-$ равновесная плотность внутренней энергии ионов, кинетические коэффициенты $C_{t o t}^{e \rightarrow b}$ и $C_{t o t}^{b \rightarrow e}$ вычисляются в задаче «К» осреднением скоростей безызлучательных переходов по распределению ионов.

Скорость обмена энергией между внутренней энергией ионов и излучением:

$$
\begin{array}{rc}
Q^{b \rightarrow r}=c \kappa_{t o t}^{b \rightarrow r} U^{P l}-c \kappa_{t o t}^{r \rightarrow b} U, & U^{P l}=\frac{4 \sigma T_{e}^{4}}{c}, \\
\kappa_{t o t}^{b \rightarrow r}=\frac{\sum_{g, n} \kappa_{g}^{b \rightarrow r,(n)} T_{g}^{(n)} U_{g}^{P l(n)}}{\sum_{g, n} T_{g}^{(n)} U_{g}^{P l(n)}}, & \kappa_{t o t}^{r \rightarrow b}=\frac{\sum_{g, n} \kappa_{g}^{r \rightarrow b,(n)} T_{g}^{(n)} U_{g}^{(n)}}{\sum_{g, n} T_{g}^{(n)} U_{g}^{(n)}} .
\end{array}
$$

Здесь $U^{P l}$ - плотность энергии излучения абсолютно черного тела, средние макросечения $\kappa_{t o t}^{b \rightarrow r}$ и $\kappa_{t o t}^{r \rightarrow b}$ вычисляются задачах «K» и «I» осреднением скоростей радиационных переходов по распределению ионов и спектральным моментам распределения фотонов на носителях резонансов.

Скорость обмена энергией между свободными электронами и полем излучения:

$$
\begin{aligned}
Q^{e \rightarrow r} & =c \kappa_{t o t}^{e \rightarrow r} U^{P l}-c \kappa_{t o t}^{r \rightarrow e} U, \\
\kappa_{t o t}^{e \rightarrow r}= & \frac{\sum_{g, n} \kappa_{g}^{e \rightarrow r,(n)} T_{g}^{(n)} U_{g}^{P l(n)}}{\sum_{g, n} T_{g}^{(n)} U_{g}^{P l(n)}}, \quad \kappa_{t o t}^{r \rightarrow e}=\frac{\sum_{g, n} \kappa_{g}^{r \rightarrow e,(n)} T_{g}^{(n)} U_{g}^{(n)}}{\sum_{g, n} T_{g}^{(n)} U_{g}^{(n)}} .
\end{aligned}
$$

Концентрация свободных электронов и средний заряд ионов вычисляются из условия квазинейтральности (4), которое записывается в виде функции от внутренней энергии ионов:

$$
N_{e}=\sum_{A, k} Z_{k}^{A} N_{k}^{A}=\sum_{A, k} \frac{Z_{k}^{A}}{E_{k}^{A}} E_{k}^{A} N_{k}^{A}=\left\langle\frac{Z}{E}\right\rangle \frac{\rho \varepsilon_{b}}{Y}, \quad\langle Z\rangle=\frac{N_{e}}{N_{a}}=\left\langle\frac{Z}{E}\right\rangle \frac{\rho \varepsilon_{b}}{Y N_{a}},
$$


где кинетический коэффициент $\langle Z / E\rangle$ вычисляется в задаче «К» по распределению ионов:

$$
\langle Z / E\rangle=\sum_{A, k} Z_{k}^{A} N_{k}^{A} / \sum_{A, k} E_{k}^{A} N_{k}^{A} .
$$

Задание расчетных объектов, сеток, «внешних» источников, краевых и начальных условий

\section{Моделирование радиационно-столкновительной кинетики и динамики плазмы}

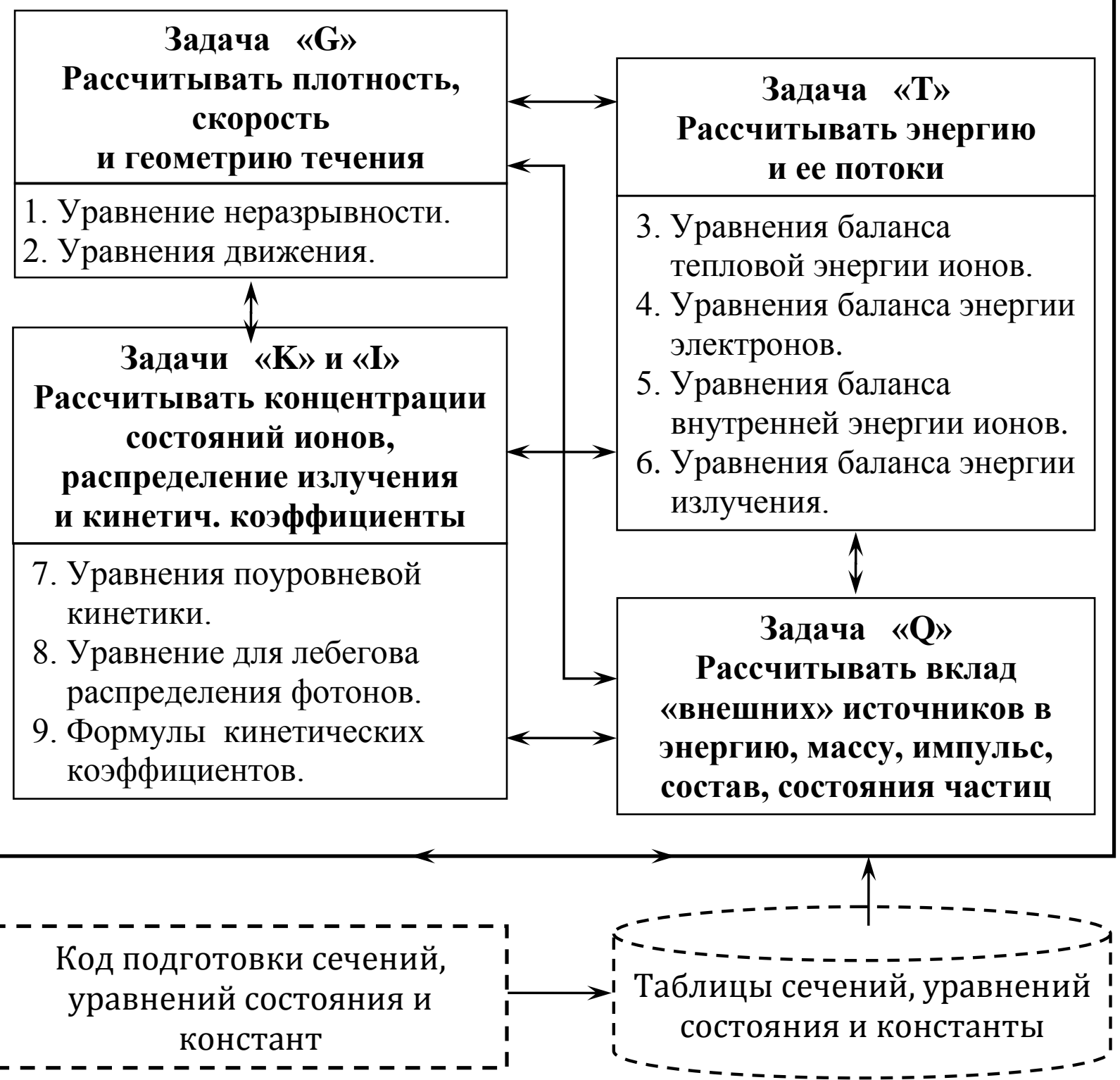

Рис. 8. Примерная методика моделирования радиачионно-столкновительной плазмы 
Скорость обмена импульсом вещества и излучения в уравнениях (25):

$\int_{0}^{\infty}\left[\kappa-\kappa_{1}^{s c}\right] \mathbf{W}(E) d E=\int_{4 \pi}^{\infty} \int_{0}^{\infty}\left[\kappa-\kappa_{1}^{s c}\right] \mathbf{\Omega} I(E, \mathbf{\Omega}) d E d \boldsymbol{\Omega} \approx\left[\kappa-\kappa_{1}^{s c}\right]_{t o t}^{0} \mathbf{W}+\left[\kappa-\kappa_{1}^{s c}\right]_{t o t}^{1} U$,

где $U(\mathbf{r}, t)$ и $\mathbf{W}(\mathbf{r}, t)$ суть полные (по спектру) плотность и поток энергии излучения. Кинетические коэффициенты $\left[\kappa-\kappa_{1}^{s c}\right]_{t o t}^{0}(\mathbf{r}, t)$ и $\quad\left[\kappa-\kappa_{1}^{s c}\right]_{t o t}^{1}(\mathbf{r}, t)$ вычисляются в задаче «I» по спектральным моментам распределения фотонов:

$$
\begin{aligned}
& {\left[\kappa-\kappa_{1}^{s c}\right]_{t o t}^{0}=\frac{1}{4 \pi} \int_{4 \pi}\left[\kappa-\kappa_{1}^{s c}\right]_{t o t} d \mathbf{\Omega}, \quad\left[\kappa-\kappa_{1}^{s c}\right]_{t o t}^{1}=\frac{1}{4 \pi} \int_{4 \pi} \boldsymbol{\Omega}\left[\kappa-\kappa_{1}^{s c}\right]_{t o t} d \mathbf{\Omega},} \\
& {\left[\kappa-\kappa_{1}^{s c}\right]_{t o t}(\mathbf{\Omega}, \mathbf{r}, t)=\sum_{g, n}\left[\kappa_{g}^{a b,(n)}+\kappa_{g}^{s c,(n)}-\kappa_{1, g}^{s c,(n)}\right] T_{g}^{(n)} J_{g}^{(n)} / \sum_{g, n} T_{g}^{(n)} J_{g}^{(n)} .}
\end{aligned}
$$

При решении задачи «Т» поля газодинамической скорости, плотности, координаты ячеек сетки и кинетические коэффициенты замораживаются. В случае линеаризации уравнений в соответствующих задачах вычисляются частные производные от кинетических коэффициентов по линейным приращениям искомых величин.

Уравнение баланса полной энергии (21) для нахождения решения задачи «Т» не используется (является линейно зависимым). Уравнение может служить средством контроля за выполнением закона сохранения энергии в системе.

Входными данными для задачи «Т» являются поле плотности и скорости, геометрия ячеек (в лагранжевом описании движения), таблицы давления и кинетической энергии электронов и ионов как функций температур и плотности, внешние источники энергии и кинетические коэффициенты для вычисления скоростей обмена между компонентами плазмы. Выходными данными являются поле температур электронов и ионов, концентрация электронов, внутренняя энергия ионов, плотность и поток энергии излучения.

Задачи «K» и «I»- рассчитывать концентрации ионов в дискретных состояниях спектра внутренней энергии ионов, лебегово распределение фотонов в объеме тела и кинетические коэффициенты, восстанавливать (если требуется) детальное спектральное распределение фотонов (39). Решается система уравнений поуровневой кинетики (61) и кинетическое уравнение (57) относительно лебегова распределения фотонов, рассчитываются спектральные моменты распределения фотонов (55).

Входными данными являются плотность и скорость вещества, геометрия ячеек, температура и концентрация электронов, сечения безызлучательных переходов и лебеговы сечения радиационных переходов ионов, равновесное распределение ионов и планковское распределение по системе лебеговых множеств. Выходными данными являются неравновесные концентрации ионов 
в состояниях дискретного спектра внутренней энергии, распределение фотонов и кинетические коэффициенты.

Задача «Q» - выполнять расчет скоростей появления (исчезновения) в системе энергии, импульса, массы и компонентов газа, плазмы. В качестве «внешних» источников могут выступать высокоэнергетичные пучки излучений, ядерные и химические реакции, фазовые превращения вещества.

Внешние входные данные для компьютерного кода моделирования радиационно-столкновительной плазмы (таблицы электронного и ионного давления, равновесной внутренней энергии, равновесных распределений ионов и излучения, сечений переходов ионов) выполняет код подготовки данных.

Если вещество находится в локальном термодинамическом равновесии, то модель радиационно-столкновительной плазмы упрощается. Из нее выпадает задача «К» - задача решения системы уравнений поуровневой кинетики (61).

\section{Заключение}

Метод спектральных моментов сводит трудоемкую задачу решения уравнения переноса излучения на подробной сетке по энергии фотонов (сечения могут содержать до миллиона разрешенных резонансов) к более простой задаче решения кинетического уравнения относительно лебегова распределения фотонов в нескольких оптимальных точках по «новой» переменной спектра - мере лебеговых множеств.

Метод дает основу для построения приближенных моделей радиационностолкновительной плазмы при малых порядках $N$, для выполнения аналитических оценок и поиска зависимостей между физическими величинами в задачах, которые ранее решались только численно.

Использование метода в численных расчетах спектрального распределения фотонов может давать значительную экономию ресурсов ЭВМ. В неоднородном гетерогенном газе или плазме для достижения «инженерной» точности (порядка 20\%) требуется найти решение уравнения переноса излучения в $4 G \div 6 G$ точках условного спектра ( $4 \div 6$ значений лебеговой меры в $G$ носителях резонансов). Количество носителей резонансов оценивается как $G \leq M[\Delta Z+1] H$, где $H$ - число элементов плазмы, $\Delta Z=0,1,2, \ldots$ - число ионов одного элемента, $M=1,2, \ldots$ - число групп состояний, вводимых для описания дискретного спектра энергии одного иона ( $M=1$ соответствует двухуровневой модели). К примеру, излучение олова (см. Рис. 1) на длине волны $\lambda=13.5 \mathrm{HM}$ или в энергии $E=91.84$ э $B$ (это излучение важно для создания источников излучения для промышленной литографии) определяется ионами с зарядом $8 \leq Z \leq 12$ [55]. Если взять с запасом $\Delta Z=7$ и использовать $M=5$ групп состояний, то для нахождения спектра фотонов потребуется ввести не более чем $G \approx 40$ носителей резонансов и не более $160 \div 240$ точек условного спектра. 
Высокая точность (не ниже точности сечений взаимодействия частиц с веществом в банках оцененных данных) достигается дальнейшим увеличением числа носителей резонансов при незначительном увеличении порядка метода $N$.

Для пространственно-угловой аппроксимации уравнения переноса излучения, аппроксимации уравнений поуровневой кинетики и уравнений динамики плазмы могут использоваться известные схемы и методы.

\section{Список литературы}

[1] $\quad$. Ambartzumian. The effect of the absorption lines on the radiative equilibrium of the outer layers of the stars // Ученые записки Ленингр. университета, серия Мат. наук (Астрономия), 1936, № 6, вып.1, с.7-18.

[2] К.Я. Кондратьев. Перенос длинноволнового излучения в атмосфере. M.: Гостехиздат, 1950. - 288 c. URL: http://www.twirpx.com/file/1346993/

[3] J.C. Stewart. Non-grey radiative transfer // J. Quant. Spectrosc. and Radiat. Transfer, 1964, v.4, p.723-729. URL: http://www.sciencedirect.com/science/article/pii/0022407364900329

[4] М.Н. Николаев, А.А. Игнатов, Н.В. Исаев и др., Метод подгрупп для учета резонансной структуры сечений в нейтронных расчетах. Часть 1 // Атомная энергия, 1970, т.29, №1, c.11-16. URL: http://link.springer.com/article/10.1007/BF01338769

[5] М.Н. Николаев, А.А. Игнатов, Н.В. Исаев и др., Метод подгрупп для учета резонансной структуры сечений в нейтронных расчетах. Часть 2 // Атомная энергия, 1971, т.30, №5, c.426-430. URL: http://link.springer.com/article/10.1007/BF01408755

[6] В.Я. Гольдин, Б.Н. Четверушкин. Методы решения одномерных задач радиационной газовой динамики // Ж. Вычисл. матем. и мат. физики, 1972, т.12, №4, c.990-1001. URL:

http://www.mathnet.ru/php/archive.phtml?wshow=paper\&jrnid=zvmmf\&paperid=6661

[7] D.E. Cullen. Application of the probability tabel method to multigroup calculations of neutron transport // J. Nuclear Science and Engineering, 1974, v.55, p.387-400. URL: http://www.ans.org/pubs/journals/nse/a 23472

[8] D.E. Cullen and G.C. Pomraning. The multiband method in radiative transfer calculations // J. Quant. Spectrosc. and Radiat. Transfer, 1980, v.24, p.97-117. URL: http://www.sciencedirect.com/science/article/pii/0022407380900242

[9] G.L. Stephens. The parameterization of radiation for numerical weather prediction and climate models // Monthly Weather Review, 1984, v.112, p.826-867. URL: http://journals.ametsoc.org/toc/mwre/112/4

[10] W.C. Wang, G.Y. Shi. Total band absorptance and k-distribution function for atmospheric gases // J. Quant. Spectrosc. and Radiat. Transfer. 1988, v.39, №5, 
p.387-397. URL: http://www.sciencedirect.com/science/article/pii/0022407388901045

[11] R.M. Goody and Y.L. Yung, Atmospheric radiation. Theoretical basis. -2 ed. New York: Oxford University Press, 1989. - 536 p.

[12] A.A. Lacis and V. Oinas, A description of the correlated k-distribution method for modeling nongray gaseous absorption. Thermal emission, and multiple scattering in vertically inhomogeneous atmospheres // J. Geophysical Research, 1991, v.96, №D5, p. 9027-9063. URL:

http://agupubs.onlinelibrary.wiley.com/agu/issue/10.1002/jgrd.v96.D5

[13] M.F. Modest. The weighted-sum-of-gray-gases model for arbitrary solution methods in radiative transfer // ASME Journal of Heat Transfer, 1991, v.113, №3, p.650-656. URL:

http://heattransfer.asmedigitalcollection.asme.org/article.aspx?articleID=1440795

[14] И.Л. Цветкова, А.В. Шильков. Осреднение уравнения переноса в резонансно поглощающей среде // Матем. моделирование, 1989, т.1, №1, c.91-100. URL:

http://www.mathnet.ru/php/archive.phtml?wshow=paper\&jrnid=mm\&paperid=2504

[15] A.B. Шильков. Методы осреднения сечений и энергетического спектра в задачах переноса нейтронов // Матем. моделирование, 1991, т.3, №2, с.6381. URL:

http://www.mathnet.ru/php/archive.phtml?wshow=paper\&jrnid=mm\&paperid=2189

[16] A.V. Shilkov. Generalized multigroup approximation and lebesgue averaging method in particle transport problems // Transp. Theory and Stat. Physics. 1994, v.23, No 6, p.781-814. URL: http://www.tandfonline.com/toc/ltty20/23/6, URL: http://arch.neicon.ru/xmlui/handle/123456789/1697260

[17] P. Riviere, A. Soufiani, J. Taine. Correlated-k and Fictitious Gas Methods for H2O near $2.7 \mu \mathrm{m} / /$ J. Quant. Spectrosc. and Radiative Transfer, 1992, v.48, p.187-203. URL: http://www.sciencedirect.com/science/article/pii/002240739290088L

[18] С.Д. Творогов. Применение рядов экспонент для интегрирования уравнения переноса по частоте // Оптика атмосферы и океана, 1999, т.12, №9, c.763-766. URL: http://ao.iao.ru/ru/content/vol.12-1999/iss.09/1

[19] А.В. Шильков, М.Н. Геризев, Верификация метода лебеговского осреднения // Матем. моделирование, 2015, том 27, №8, c.13-31. URL: http://www.mathnet.ru/php/archive.phtml?wshow=paper\&jrnid=mm\&paperid=3635

[20] С.В. Можейко, И.Л. Цветкова, А.В. Шильков. Расчет переноса излучения в горячем воздухе // Матем. моделирование, 1992, т.4, №1, с.65-82. URL: http://www.mathnet.ru/php/archive.phtml? wshow=paper\&jrnid=mm\&paperid=2035

[21] А.В. Шильков, И.Л. Цветкова, С.В. Шилькова. Система ATRAD для расчетов атмосферной радиации: лебеговское осреднение спектров и сечений поглощения // Матем. моделирование, 1997, т.9, №6, с.3-24. URL: http://www.mathnet.ru/php/archive.phtml?wshow=paper\&jrnid=mm\&paperid=1421

[22] Ю.Д. Шмыслевский, Расчет переноса лучистой энергии методом Галеркина // Вычисл. матем. и мат. физики, 1973, том 13, № 2, с.398-407. http://www.mathnet.ru/php/archive.phtml?wshow=paper\&jrnid=zvmmf\&paperid=8354 
[23] Ю.Д. Шмыллевский, Вариант моментного метода расчета переноса селективного излучения. // Вычисл. матем. и мат. физики, 1977, том 17, №3, c. 785-790. URL: http://www.mathnet.ru/php/archive.phtml?wshow=paper\&jrnid=zvmmf\&paperid=5977

[24] В.М. Кривцов. Об одном подходе к расчету селективного излучения // Вычисл. матем. и мат. физики, 1974, т. 14, № 6, с. 1595-1599. URL: http://www.mathnet.ru/php/archive.phtml? wshow=paper\&jrnid=zvmmf\&paperid=9395

[25] В.М. Кривцов. О расчете селективного излучения. В сб.: «Динамика излучающего газа, вып.2», ред. Ю.Д. Шмыглевский. М.: Изд-во ВЦ АН CCCP, 1976, с. 36-41.

[26] Я.Б. Зельдович, Ю.П. Райзер. Физика ударных волн и высокотемпературных гидродинамических явлений. - М.: Наука, 1966. URL: http://www.twirpx.com/file/46551/

[27] G.C. Pomraning. The equations of radiation hydrodynamics. - New York: Pergamon Press, 1973. - 298 pp.

[28] Л.М. Биберман, В.С. Воробьев, И.Т. Якубов. Кинетика неравновесной низкотемпературной плазмы. - М.: Наука, 1982. - 378 c. URL: http://www.twirpx.com/file/216964/

[29] А.Н. Ключарев, Н.Н. Безуглов. Процессы возбуждения и ионизации атомов при поглощении света. - Ленинград: Изд-во Ленингр. ун-та, 1983.

[30] В.И. Держиев, А.Г. Жидков, С.И. Яковленко. Излучение ионов в неравновесной плотной плазме. - М.: Энергоатомиздат, 1986. - 160 с. URL: http://www.twirpx.com/file/1188180/

[31] Д.И. Нагирнер. Лекции по теории переноса излучения. - СПб.: Изд-во С.Петерб. ун-та, 2001. - 284 c. URL: http://www.twirpx.com/file/367042/

[32] В.С. Имшенник, Ю.И. Морозов. Радиационная релятивистская газодинамика высокотемпературных явлений. - М.: Атомиздат, 1981. - 88 c. URL: http://www.twirpx.com/file/462994/

[33] Д. Михалас. Звездные атмосферы, том 1 и 2. - Москва: Mир, 1982. URL: http://www.twirpx.com/file/39098/ URL: http://www.twirpx.com/file/543852/

[34] D. Mihalas, B.W. Mihalas. Foundations of radiation hydrodynamics. - New York: Oxford University Press, 1984. - 730 pp. URL: http://www.fas.org/sgp/othergov/doe/lanl/index $1 . \mathrm{html}$

[35] A. Peraiah. An introduction to radiative transfer. - Cambridge, UK: Cambridge University Press, 2004. - 494 pp.

[36] J.I. Castor. Radiation hydrodynamics. - Cambridge, New York: Cambridge University Press, 2004. - 368 pp.

[37] W. Kalkofen, (ed.) Methods in radiative transfer. - New York, London: Cambridge University Press, 1984. - 456 pp.

[38] W. Kalkofen, (ed.) Numerical radiative transfer. - New York, London: Cambridge University Press, 1988. - 382 pp.

[39] Y. Ralchenko. (ed.) Modern methods in collisional-radiative modeling of 
plasmas. - Switzerland: Springer Int. Publ., 2016. - 218 pp. URL: http://link.springer.com/book/10.1007/978-3-319-27514-7

[40] Л. Спитиер. Физика полностью ионизованного газа. - Пер. с англ. М.: Миp, 1965. - 212 c. URL: http://www.twirpx.com/file/158500/

[41] С.И. Брагинский Явления переноса в плазме // В кн. «Вопросы теории плазмы, вып. 1», с. 183 - 272. Под ред. М.А. Леонтовича. - М:

Госатомиздат, 1963. URL: http://www.twirpx.com/file/17157/

[42] В.П. Силин Введение в кинетическую теорию газов. - М: Наука, 1971. URL: http://www.twirpx.com/file/583590/

[43] Теория нагрева и сжатия низкоэнтропийных термоядерных мишеней. Труды ФИАН, том. 134, под редакцией Н.Г. Басова. - М.: Наука, 1982. URL: http://www.twirpx.com/file/1507307/

[44] Д. Бейтс (ред.) Атомные и молекулярные процессы. - Пер. с англ. М.: Мир, 1964. - 777 с.

[45] И.И. Собельман. Введение в теорию атомных спектров. - М.: ГИФМЛ, 1963. - 640 c. URL: http://www.twirpx.com/file/261678/

[46] В.А. Вайнштейн, И.И. Собельман, Е.А. Юков. Возбуждение атомов и уширение спектральных линий. - М.: Наука, 1979. URL: http://www.twirpx.com/file/1125326/

[47] R.D. Cowan. The theory of atomic structure and spectra. - Los Angeles, London: University of California Press, 1981. - 731.

[48] В.П. Жданов. Диэлектронная рекомбинация // В кн. «Вопросы теории плазмы, т. 12», под.ред. М.А .Леонтовича и Б.Б. Кадомцева. - М.: Энергоиздат. 1982. c.79-93. URL: http://www.twirpx.com/file/78904/

[49] D. Salzmann. Atomic physics in hot plasmas. - New York, Oxford: Oxford University Press, 1998.

[50] В.Г. Новиков, А.Д. Соломянная. Спектральные характеристики плазмы, согласованные с излучением // TBT, 1998, т. 36, Вып. 6, с. 858-864. URL: http://www.mathnet.ru/php/archive.phtml?wshow=paper\&jrnid=tvt\&paperid=2658

[51] А.Ф. Никифоров, В.Г. Новиков, В.Б. Уваров. Квантово-статистические модели высокотемпературной плазмы и методы расчета росселандовых пробегов и уравнений состояния. - М.: Физматлит, 2000. - 400 c. URL: http://www.twirpx.com/file/552022/

[52] A.F. Nikiforov, V.G. Novikov, and V.B. Uvarov. Quantum-statistical models of hot dense matter: methods for calculation opacity and equation of state. Basel-Boston-Berlin: Birkhauser, 2005.

[53] В.Г. Новиков, А.Д. Соломянная, В.С. Захаров. Квантово-статистические методы расчета оптических и термодинамических свойств плотной плазмы. В кн. «Энц. низкотемп. плазмы. Сер.Б, т. 7-1, ч.2 (7-1/2): Матем. моделирование в низкотемп. плазме». М.: Янус К. 2008. с. 373-435.

[54] Л.М. Биберман, И.Т. Якубов. Состояние газа за фронтом сильной ударной волны // Теплофизика высоких температур, 1965, т. 3, № 3, с. 340-353. 
URL: http://www.mathnet.ru/php/archive.phtml?wshow=paper\&jrnid=tvt\&paperid=4045

[55] Д.А. Ким, В.Г. Новиков, Г.В. Долголева, К.Н. Комелев, А.Д. Соломянная. Моделирование источников EUV-излучения с учетом детальной кинетики, включенной in-line в программу радиационной газодинамики // Матем. моделирование, 2013, т. 25, № 7, с. 89-102. URL: http://www.mathnet.ru/php/archive.phtml?wshow=paper\&jrnid=mm\&paperid=3384

[56] C. Чандрасекар. Перенос лучистой энергии. - Пер. с англ. М.: Иностр. литература, 1953. - 432 c. URL: http://www.twirpx.com/file/195970/

[57] Б.Г. Карлсон, К.Д. Латроп, Теория переноса - метод дискретных ординат. - Пер. с англ. В сб. «Вычислительные методы в физике реакторов», под ред. Х. Гринспена, К. Келбера, Д. Окрента / М.: Атомиздат, 1972, с.102157. URL: http://www.twirpx.com/file/655947/

[58] Б. Дэвисон. Теория переноса нейтронов. - Пер. с англ. М.: Атомиздат, 1960. - 520 c. URL: http://www.twirpx.com/file/999788/

[59] В.Я. Гольдин. Квазидиффузионный метод решения кинетического уравнения // Ж. Вычисл. матем. и мат. физики. 1964, т. 4, № 6, с. 10781087. URL: http://www.mathnet.ru/php/archive.phtml?wshow=paper\&jrnid=zvmmf\&paperid=7676

[60] Б.Н. Четверушкин. Математическое моделирование задач динамики излучающего газа. - М.: Наука, 1985. - 304 с. URL: http://www.twirpx.com/file/1329349/

[61] В.Я. Гольдин, Д.А. Гольдина, А.В. Колпаков, А.В. Шильков. Математическое моделирование газодинамических процессов при высокой плотности энергии излучения // ВАНТ, Серия Методики и прогр. числ. решения задач мат. физики, 1986, вып. 2, с. 59 - 66.

[62] E.H. Аристова. Моделирование взаимодействия излучения с веществом. Применение метода квазидиффузии. - Saarbrucken: Изд-во Lambert, Academic Publishing, 2011. - 297 c.

[63] С.T. Суржиков. Тепловое излучение газов и плазмы. - М: Изд-во МГТУ им. Н.Э. Баумана, 2004. -544 с.

[64] C.T. Суржиков. Радиационная газовая динамика спускаемых космических аппаратов. Многотемпературные модели. - М: ИПМех им. А.Ю. Ишлинского PAH, 2013. - 706 с. URL: http://www.twirpx.com/file/1751365/

[65] А.В. Шильков. Уравнения для спектральных моментов функции распределения фотонов // М.: Препринты ИПМ им. М.В. Келдыша. 2015, №77, 36 c. URL: http://library.keldysh.ru/preprint.asp?id=2015-77

[66] Г. Сеге, Ортогональные многочлены. - М.: Физматлит, 1962. - 500 c. URL: http://www.twirpx.com/file/493859/

[67] W.M. Elsasser. Mean absorption and equivalent absorption coefficient of a band spectrum // Physical Review, 1938, v.54, p.126. URL: http://journals.aps.org/pr/abstract/10.1103/PhysRev.54.126 


\section{Константы. Обозначения величин}

Константы

\begin{tabular}{|c|c|}
\hline Число Авогадро & $N_{A \nu}=6.02214129 \cdot 10^{23}$ моль $^{-1}$ \\
\hline $\begin{array}{l}\text { Масштабный } \\
\text { множитель перевода } \\
\text { единиц энергии }\end{array}$ & $\begin{array}{l}\text { из электронвольт в эрг: } Y=1.602176565 \cdot 10^{-12}{ }_{э р г} \cdot{ } B^{-1} \\
\text { из электронвольт в градус: } Y / k=1.16045193 \cdot 10^{4} K \cdot{ }^{-1}\end{array}$ \\
\hline $\begin{array}{l}\text { Постоянная } \\
\text { Стефана-Больцмана } \\
U_{\left[e r g \cdot c m^{-3}\right]}^{P l}=4 \sigma T^{4} / c\end{array}$ & $\begin{array}{l}\sigma=2 \pi^{5} k^{4} / 15 c^{2} h^{3}=5.670373 \cdot 10^{-5} э р 2 \cdot c е \kappa^{-1} c M^{-2} K^{-4} \\
\sigma=2 \pi^{5} Y^{4} / 15 c^{2} h^{3}=1.028301 \cdot 10^{12} э р г \cdot c e \kappa^{-1} c M^{-2} \ni B^{-4}\end{array}$ \\
\hline
\end{tabular}

\section{Обозначения величин}

\begin{tabular}{|c|c|}
\hline$\langle A\rangle_{[г / \text { моль }]}$ & Средний атомный вес ионов. \\
\hline$\overline{A, n}$ & Состояние иона с внутренней энергией $E_{n}^{A}$. \\
\hline$A, k$ & $\begin{array}{l}\text { Группа состояний иона со средней внутренней } \\
\text { энергией } E_{k}^{A} \text {. }\end{array}$ \\
\hline$C_{n \rightarrow m,\left[\text { ceK }^{-1}\right]}^{A}$ & $\begin{array}{l}\text { Суммарная скорость безызлучательных переходов } \\
\text { иона из состояния с энергией } E_{n}^{A} \text { в состояние с } \\
\text { большей энергией } E_{m}^{A} \text {. }\end{array}$ \\
\hline$\frac{d}{d l}=\frac{1}{c} \frac{\partial}{\partial t}+\mathbf{\Omega} \frac{\partial}{\partial \mathbf{r}}$ & $\begin{array}{l}\text { Полная производная вдоль траектории движения } \\
\text { пучка фотонов (характеристики). }\end{array}$ \\
\hline$\frac{d}{d t}=\frac{\partial}{\partial t}+\mathbf{u} \frac{\partial}{\partial \mathbf{r}}$ & $\begin{array}{l}\text { Субстанциональная производная (substance (англ.) - } \\
\text { вещество). }\end{array}$ \\
\hline$E_{[\ni B]},(E=h v)$ & Энергия фотона в сопутствующей системе отсчета. \\
\hline$E \mathbf{\Omega} / c$ & Испульс фотона в сопутствующей системе отсчета. \\
\hline$E_{n,[\ni B]}^{A}$ & Внутренняя энергия иона в состоянии $A, n$. \\
\hline 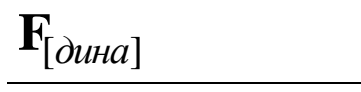 & Внешняя сила. \\
\hline$g_{n}^{A}$ & $\begin{array}{l}\text { Статистический вес энергетического состояния иона } \\
A, n .\end{array}$ \\
\hline$I_{\left[э р г \cdot с \mathrm{c}^{-2}{ }{ }^{-1} \kappa^{-1}{ }^{-1} B^{1}\right]}$ & $\begin{array}{l}\text { Интенсивность излучения или функции распределения } \\
\text { фотонов. }\end{array}$ \\
\hline$I_{\left[э p 2 \cdot c M^{-2}{ }^{2} e \kappa^{-1}{ }^{-1} B^{-1}\right]}^{\text {ext }}$ & $\begin{array}{l}\text { Интенсивность «внешнего» излучения, прошедшего } \\
\text { сквозь границу (испущенного границей) вглубь тела. }\end{array}$ \\
\hline
\end{tabular}




\begin{tabular}{|c|c|}
\hline$N_{a,\left[c M^{-3}\right]}$ & Концентрация ионов (атомов и ионов). \\
\hline$N_{A,\left[\mathrm{CM}^{-3}\right]}$ & Концентрация ионов с атомным весом $A$. \\
\hline$N_{e,\left[c M^{-3}\right]}$ & Концентрация свободных электронов. \\
\hline$N_{n,\left[c M^{-3}\right]}^{A}$ & Концентрация ионов в состоянии $A, n$ с энергией $E_{n}^{A}$. \\
\hline $\bar{N}_{n,\left[\mathrm{CM}^{-3}\right]}^{A}$ & $\begin{array}{l}\text { Концентрация ионов в состоянии } A, n \text { с энергией } E_{n}^{A} \\
\text { при локальном термодинамическом равновесии. }\end{array}$ \\
\hline$P_{e,\left[э р г \cdot \mathcal{C M}^{-3}\right]}$ & Давление газа свободных электронов. \\
\hline$P_{a,\left[э р г \cdot \mathrm{cm}^{-3}\right]}$ & Давление газа ионов. \\
\hline$P_{i j,\left[э р г \cdot c M^{-3}\right]}^{r}$ & $\begin{array}{l}\text { Тензор давления излучения в (сопутствующей) } \\
\text { системе отсчета, движущейся с жидкой частицей. }\end{array}$ \\
\hline$P_{i j}^{r}(E)_{\left[э p 2 \cdot c M^{-3} \ni B^{-1}\right]}$ & $\begin{array}{l}\text { Распределение тензора давления излучения по спектру } \\
\text { энергий фотонов в сопутствующей системе отсчета. }\end{array}$ \\
\hline$Q_{e,\left[э р г \cdot с е \kappa^{-1} \mathrm{~cm}^{-3}\right]}$ & $\begin{array}{l}\text { Внешний объемный источник поступления энергии в } \\
\text { газ электронов. }\end{array}$ \\
\hline$Q_{a,\left[э р г \cdot с е к^{-1} с^{-3}\right]}$ & $\begin{array}{l}\text { Внешний объемный источник поступления энергии в } \\
\text { газ ионов. }\end{array}$ \\
\hline$Q_{\left[э р 2 \cdot{ }^{2} е \kappa^{-1} \mathrm{CM}^{-3}\right]}^{e \rightarrow a}$ & $\begin{array}{l}\text { Скорость обмена энергией между электронами и } \\
\text { хаотическим движением ионов. }\end{array}$ \\
\hline$Q_{\left\lceil э р 2 \cdot \operatorname{ce\kappa }^{-1} \mathrm{cM}^{-3}\right]}^{e \rightarrow b}$ & $\begin{array}{l}\text { Обмен энергией между электронами и внутренней } \\
\text { энергией ионов в безызлучательных столкновениях. }\end{array}$ \\
\hline$Q_{\left[э р 2 \cdot{ }^{2} е \kappa^{-1} \mathrm{CM}^{-3}\right]}^{e \rightarrow r}$ & $\begin{array}{l}\text { Скорость обмена энергией между электронами и } \\
\text { полем излучения. }\end{array}$ \\
\hline$Q_{\left[э р 2 \cdot \mathrm{ce \kappa}^{-1} \mathrm{cM}^{-3}\right]}^{b \rightarrow r}$ & $\begin{array}{l}\text { Скорость обмена энергией между внутренней } \\
\text { энергией ионов и полем излучения. }\end{array}$ \\
\hline$T_{e,[э B]}$ & Температура свободных электронов. \\
\hline$T_{a,[\ni B]}$ & Температура хаотического движения ионов. \\
\hline $\mathbf{u}_{[c \mathrm{~cm} / с е \kappa]}$ & Газодинамическая скорость плазмы. \\
\hline$U(E)_{\left[э р ح \cdot c M^{-3} э B^{-1}\right]}$ & $\begin{array}{l}\text { Распределение плотности энергии излучения по } \\
\text { спектру в сопутствующей системе отсчета. }\end{array}$ \\
\hline$U_{\left[э р г \cdot с M^{-3}\right]}$ & $\begin{array}{l}\text { Плотность энергии излучения в сопутствующей } \\
\text { системе отсчета, связанной с жидкой частицей. }\end{array}$ \\
\hline$U^{P l}\left(E, T_{e}\right)_{\left[э р г \cdot \mathrm{CM}^{-3} э B^{-1}\right]}$ & Распределение Планка. \\
\hline$U_{\left[э р ح \cdot c M^{-3}\right]}^{P l}=4 \sigma T_{e}^{4} / c$ & Плотность энергии излучения абсолютно чер \\
\hline
\end{tabular}




\begin{tabular}{|c|c|}
\hline $\mathbf{W}(E)_{\left[э р 2 \cdot c M^{-2} c e \kappa^{-1} \ni B^{-1}\right]}$ & $\begin{array}{l}\text { Распределение потока энергии излучения по спектру в } \\
\text { сопутствующей системе отсчета. }\end{array}$ \\
\hline $\mathbf{W}_{\left[э р г \cdot c M^{-2} c е \kappa^{-1}\right]}$ & Поток энергии излучения в сопутствующей системе. \\
\hline $\mathbf{W}_{\left[э р г \cdot с \mathcal{M}^{-2} \text { сек }^{-1}\right]}^{e}$ & Поток электронной теплопроводности. \\
\hline $\mathbf{W}_{\left[э р г \cdot с \mathcal{M}^{-2} \text { сек }^{-1}\right]}^{a}$ & Поток ионной теплопроводности. \\
\hline$\langle Z\rangle,\langle Z\rangle_{A}$ & $\begin{array}{l}\text { Средний заряд ионов и средний заряд ионов с } \\
\text { атомным весом } A \text {. }\end{array}$ \\
\hline$\overline{Z_{n}^{A}}$ & Заряд иона в энергетическом состоянии $A, n$. \\
\hline
\end{tabular}

\begin{tabular}{|c|c|}
\hline$\varepsilon_{e,[э р г / 2]}$ & Удельная энергия хаотического движения электронов. \\
\hline$\varepsilon_{a,[э р г / 2]}$ & Удельная энергия хаотического движения ионов. \\
\hline$\varepsilon_{b,[э р г / 2]}$ & Удельная внутренняя энергия ионов. \\
\hline$\kappa_{\left[c M^{-1}\right]}=\kappa^{a b}+\kappa^{s c}$ & Макросечение экстинкции фотонов. \\
\hline$\kappa_{\left[c M^{-1}\right]}^{s c}, K^{s c}\left(\mathbf{\Omega}^{\prime}\right)_{\left[c M^{-1}\right]}$ & $\begin{array}{l}\text { Макросечение рассеяния (scattering - англ.) и } \\
\text { дифференциальное сечение рассеяния излучения. }\end{array}$ \\
\hline$\kappa_{\left[c M^{-1}\right]}^{a b}$ и $\kappa_{\left[c M^{-1}\right]}^{e m}$ & $\begin{array}{l}\text { Макросечение поглощения (absorption - англ.) и } \\
\text { макросечение испускания (emission - англ.) излучения. }\end{array}$ \\
\hline$\kappa_{\left[c M^{-1}\right]}^{r \rightarrow b}$ и $\kappa_{\left[c M^{-1}\right]}^{b \rightarrow r}$ & $\begin{array}{l}\text { Макросечение поглощения и макросечение } \\
\text { испускания энергии излучения ионами. }\end{array}$ \\
\hline$\kappa_{\left[c M^{-1}\right]}^{r \rightarrow e}$ и $\kappa_{\left[c M^{-1}\right]}^{e \rightarrow r}$ & $\begin{array}{l}\text { Макросечение поглощения и макросечение } \\
\text { испускания энергии излучения электронами. }\end{array}$ \\
\hline$\rho_{\left[2 / c M^{3}\right]}$ & Плотность плазмы. \\
\hline$\sigma_{n \rightarrow m,\left[c m^{2}\right]}^{A}$ & $\begin{array}{l}\text { Суммарное сечение переходов иона из состояния с } \\
\text { энергией } E_{n}^{A} \text { в состояние с большей энергией } E_{m}^{A} \text {. }\end{array}$ \\
\hline$\sigma_{m \rightarrow n,\left[\mathrm{~cm}^{2}\right]}^{A}$ & $\begin{array}{l}\text { Суммарное сечение обратных переходов иона из } \\
\text { состояния с энергией } E_{m}^{A} \text { в состояние с } E_{n}^{A}<E_{m}^{A} \text {. }\end{array}$ \\
\hline$\sigma_{i j,\left[э р г \cdot \mathrm{cm}^{-3}\right]}^{e}, \sigma_{i j,\left[э р г \cdot \mathrm{cm}^{-3}\right]}^{a}$ & Тензоры вязкости свободных электронов и ионов. \\
\hline$\Sigma\left(E, \boldsymbol{\Omega}^{\prime} \rightarrow \boldsymbol{\Omega}, \mathbf{r}_{i n}, t\right)$ & Сечение отражения излучения от границы тела. \\
\hline$\chi_{e}, \chi_{a}$ & $\begin{array}{l}\text { Коэффициенты электронной и ионной } \\
\text { теплопроводности. }\end{array}$ \\
\hline $\mathbf{\Omega},(|\mathbf{\Omega}|=1)$ & $\begin{array}{l}\text { Единичный вектор-направление полета пучка фотонов } \\
\text { в сопутствующей системе отсчета. }\end{array}$ \\
\hline
\end{tabular}

